\title{
Social Media and mHealth Technology for Cancer Screening: Systematic Review and Meta-analysis
}

Arlinda Ruco ${ }^{1,2}$, MPH; Fahima Dossa ${ }^{3}$, MD; Jill Tinmouth ${ }^{1,4,5}$, MD, PhD, FRCPC; Diego Llovet ${ }^{1,4}$, PhD; Jenna Jacobson $^{1,6}$, PhD; Teruko Kishibe ${ }^{7}$, MISt; Nancy Baxter ${ }^{1,2,8}$, MD, PhD, FRCSC

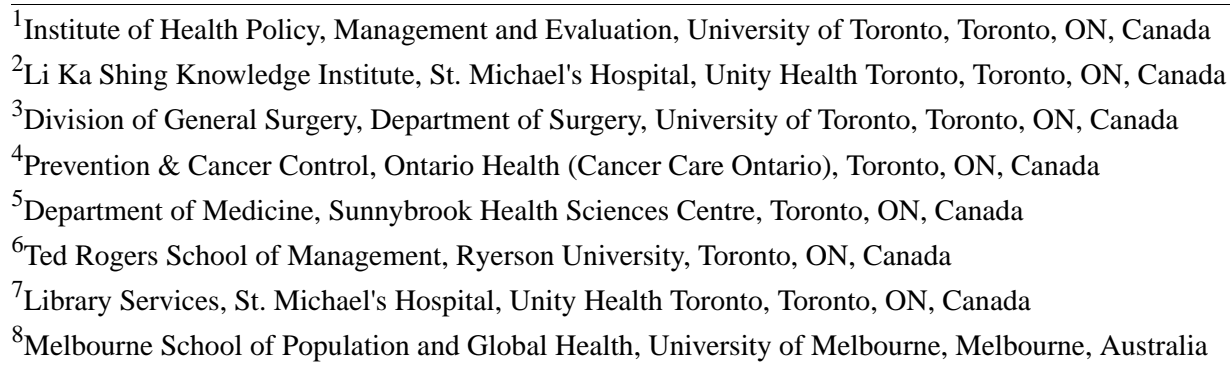

Corresponding Author:

Nancy Baxter, MD, PhD, FRCSC

Melbourne School of Population and Global Health

University of Melbourne

207 Bouverie Street

Melbourne, 3053

Australia

Phone: 6139035551

Email: Nancy.baxter@unimelb.edu.au

\section{Abstract}

Background: Cancer is a leading cause of death, and although screening can reduce cancer morbidity and mortality, participation in screening remains suboptimal.

Objective: This systematic review and meta-analysis aims to evaluate the effectiveness of social media and mobile health (mHealth) interventions for cancer screening.

Methods: We searched for randomized controlled trials and quasi-experimental studies of social media and mHealth interventions promoting cancer screening (breast, cervical, colorectal, lung, and prostate cancers) in adults in MEDLINE, Embase, PsycINFO, Scopus, CINAHL, Cochrane Central Register of Controlled Trials, and Communication \& Mass Media Complete from January 1, 2000, to July 17, 2020. Two independent reviewers screened the titles, abstracts, and full-text articles and completed the risk of bias assessments. We pooled odds ratios for screening participation using the Mantel-Haenszel method in a random-effects model.

Results: We screened 18,008 records identifying 39 studies (35 mHealth and 4 social media). The types of interventions included peer support $(n=1)$, education or awareness $(n=6)$, reminders $(n=13)$, or mixed $(n=19)$. The overall pooled odds ratio was 1.49 (95\% CI 1.31-1.70), with similar effect sizes across cancer types.

Conclusions: Screening programs should consider mHealth interventions because of their promising role in promoting cancer screening participation. Given the limited number of studies identified, further research is needed for social media interventions.

Trial Registration: PROSPERO International Prospective Register of Systematic Reviews CRD42019139615; https://www.crd.york.ac.uk/prospero/display_record.php?RecordID=139615

International Registered Report Identifier (IRRID)： RR2-10.1136/bmjopen-2019-035411

(J Med Internet Res 2021;23(7):e26759) doi: 10.2196/26759

\section{KEYWORDS}

social media; mHealth; cancer screening; digital health; mass screening; mobile phone 


\section{Introduction}

\section{Background}

The use of mobile health (mHealth) technologies and social media in the health care sphere has now become widespread [1-6] and has enabled the rapid sharing of health information, the launching of health promotional campaigns, access to peer support groups, and facilitation of appointment reminders $[1,2,4,6]$. The World Health Organization has defined mHealth as the use of mobile wireless devices for medical and public health practice [1]. Social media allows those with access to information and communication technology to become content creators and share content with others in virtual communities or networks in addition to accessing information and connecting communities $[1,6]$. The use of mHealth and social media for health presents an important opportunity to reach health consumers, as these technologies and platforms can provide more frequent interactions, deliver tailored material, and increase accessibility to health information [1], and they now constitute a major way of communicating and advertising. In addition, as access to mobile devices and the internet in low- and middle-resource nations is reported to be comparable with those in developed countries, mHealth and social media may play a role in closing the gap in health disparities between high- and low-resource nations $[1,7]$.

With almost 19 million people expected to be diagnosed with cancer in 2020, cancer is one of the leading causes of death globally [8]. Cancer screening has been shown to reduce disease-specific mortality for a number of cancers [9-12], and as a result, many jurisdictions have implemented population-based screening programs [13,14]. However, screening participation remains suboptimal across jurisdictions and cancer types [13-16]. Emerging research has explored the use of social media and mHealth for cancer screening [17-21]. However, we currently lack an understanding of how effective mHealth and social media can be for cancer screening participation.

\section{Objectives}

This systematic review and meta-analysis aims to explore the effectiveness of social media and mHealth interventions to increase cancer screening participation and intention for screen detectable cancers.

\section{Methods}

\section{Study Design and Registration}

This systematic review was registered with the International PROSPERO (Prospective Register of Systematic Reviews; registration \#CRD42019139615) and was written and reported according to the PRISMA (Preferred Reporting Items for Systematic Reviews and Meta-Analyses) checklist [22].

\section{Inclusion and Exclusion Criteria}

Studies included in this systematic review were randomized controlled trials (RCTs) or quasi-experimental studies with a pre- and postintervention design reporting on the effectiveness of an mHealth or social media intervention on cancer screening participation or intention. We included studies pertaining to breast, cervical, colorectal, prostate, or lung cancer, as guidelines for screening exist for these cancers. We defined mHealth interventions as those that delivered health-related information via telecommunication or other wireless technologies (eg, smartphones and tablets) [4]. Social media interventions included those delivered on an already established or new purpose-built social media platform where users could create a profile and share content with other users (virtual communities) [1]. Any comparator was acceptable, including a nonintervention group; an alternate, nonsocial media, non-mHealth intervention; or studies with a pre- and postintervention design. We included studies with multifaceted interventions if at least one component involved a social media- or mHealth-based strategy. Studies were restricted to those conducted in adults aged 18 years or older and articles published in English. In case we were unable to access full-text articles for relevant abstracts, we contacted study authors to obtain the articles. If the authors did not respond, we included the abstract if we could ascertain the eligibility criteria and if the data on the primary or secondary outcome were available. Commentaries, editorials, letters, and reviews were excluded. We also excluded articles published before 2000 because the use of social media was not widespread before this time [4].

\section{Search Strategy}

The search strategy was developed by a senior information specialist (TK) and used a combination of text words and MeSH (Medical Subject Headings) terms depending on the database to capture the following concepts: cancer, screening, and social media or mHealth interventions. The search strategy was peer reviewed by a second information specialist in accordance with the Peer Review of Electronic Search Strategies checklist [23] and has been previously published [24].

\section{Information Sources}

The search was conducted using the following databases: MEDLINE, Embase, PsycINFO, Scopus, CINAHL, the Cochrane Central Register of Controlled Trials, and Communication \& Mass Media Complete from inception to May 31, 2019. The search was updated on July 17, 2020.

\section{Data Management}

We used systematic review software (DistillerSR, Evidence Partners Incorporated) to manage records during the screening and study selection phases.

\section{Study Selection}

Two independent reviewers (AR and FD) used a piloted data collection form and screened the studies in three stages: title, abstract, and full text. Citations that either reviewer considered potentially eligible at the title stage were included to maximize sensitivity in the early stages of screening. Inclusion in the abstract and full-text screening stages required consensus between the reviewers. Discrepancies between the reviewers at the abstract or full-text stages were resolved by discussion.

\section{Data Extraction}

Two reviewers independently extracted data from the included studies using a piloted data collection form in Excel (Version 
15.0; Microsoft). Any discrepancies were resolved by discussion. Information extracted from each study included study characteristics (authors, date of publication, location or country, funding, and study design), participant characteristics (sample size, age, sex, ethnicity, and eligibility), intervention details (type of intervention, components, comparator or control group interventions, follow-up or duration, technology platform, and delivery of intervention by whom), and outcomes of interest (screening participation or intention including timeframe).

\section{Outcomes}

Screening participation (primary outcome) was defined as the proportion of adults who participated in the screening. This included self-reported outcomes as well as those confirmed through administrative records. Screening intention (secondary outcome) was defined as per the primary study authors. Typically, this is measured as the written intention to undergo screening within a specified timeframe (eg, within the next 3 months or 6 months).

\section{Assessment of Bias}

The Cochrane Risk of Bias 2 tool [25] was used to assess the quality of RCTs, and the Cochrane Effective Practice and Organization of Care framework was used to assess bias in preand postintervention studies [26]. The risk of bias assessment was independently completed for each study by 2 reviewers (AR and FD). Discrepancies were resolved by discussion or by a third investigator if needed. The Robvis tool was used to create a risk of bias plot [27].

\section{Data Synthesis and Analysis}

The study, participant, and intervention characteristics and the risk of bias assessments are presented descriptively. We categorized interventions based on their nature, including (1) reminders, (2) education or awareness, (3) navigation or counseling, (4) peer support, (5) decision aids, and (6) mixed. We report on the outcomes of interest in absolute and relative terms and pooled odds ratios (ORs) for screening participation from RCTs using the Mantel-Haenszel method in a random-effects model. If the outcome was measured at several time points, we used the values from the longest follow-up for our study. In RCTs where several intervention arms had a social media or mHealth component, we included them in our analysis and divided the proportion screened of the control or comparison group equally by the number of intervention arms of interest to maintain the same proportion of those screened while not counting the sample size of the control group more than once, as recommended by Cochrane [28]. Forest plots were created to graphically display results stratified by cancer type and the nature of the intervention. Statistical heterogeneity was calculated using the $\mathrm{I}^{2}$ statistic, where a cutoff of $\geq 75 \%$ was defined as considerable heterogeneity [28]. We conducted a sensitivity analysis in which we excluded articles that were assessed to have a high risk of bias. In addition, we conducted sensitivity analyses to explore whether the overall pooled effect estimate would differ for studies measuring the outcome of cancer screening participation through self-reporting compared with objective or administrative records and for studies conducted in low- and middle-income countries (LMICs). We checked for publication bias for the primary outcome among the RCTs using a funnel plot. Statistical significance was set at a two-tailed $P<.05$. Meta-analyses were performed using Review Manager (RevMan, The Cochrane Collaboration) 5.0.

\section{Results}

\section{Search Results and Characteristics of Included Studies}

A total of 18,008 records were identified in the search. After duplicates were removed, 17,788 titles, 2607 abstracts, and 687 full-text articles were screened. After all the eligibility criteria were applied, 39 articles were included [29-67] (Figure 1). Table 1 presents a summary of the included RCTs $(n=30)$, and Table 2 presents an overview of the included pre- and postintervention studies $(n=9)$. Briefly, the studies that were included were published between 2011 and 2020 and conducted in North America, Europe, Asia, and Africa. Most of the studies (35/39, $90 \%$ ) described mHealth interventions, and 10\% (4/39) of them included social media. The most common type of intervention was mixed $(n=19)$, followed by reminders $(n=13)$, education or awareness $(n=6)$, and peer support $(n=1)$. Mixed interventions were most commonly a combination of reminder and education strategies. There were 16 studies focused on cervical cancer, 14 on colorectal cancer (CRC), 7 on breast cancer, and 1 each on lung and prostate cancer screening. The interventions were implemented by public or private screening programs, university-based research teams, or health care centers or units. 
Figure 1. PRISMA (Preferred Reporting Items for Systematic Reviews and Meta-Analyses) diagram outlining the steps involved in identifying screened and included studies in the meta-analysis.

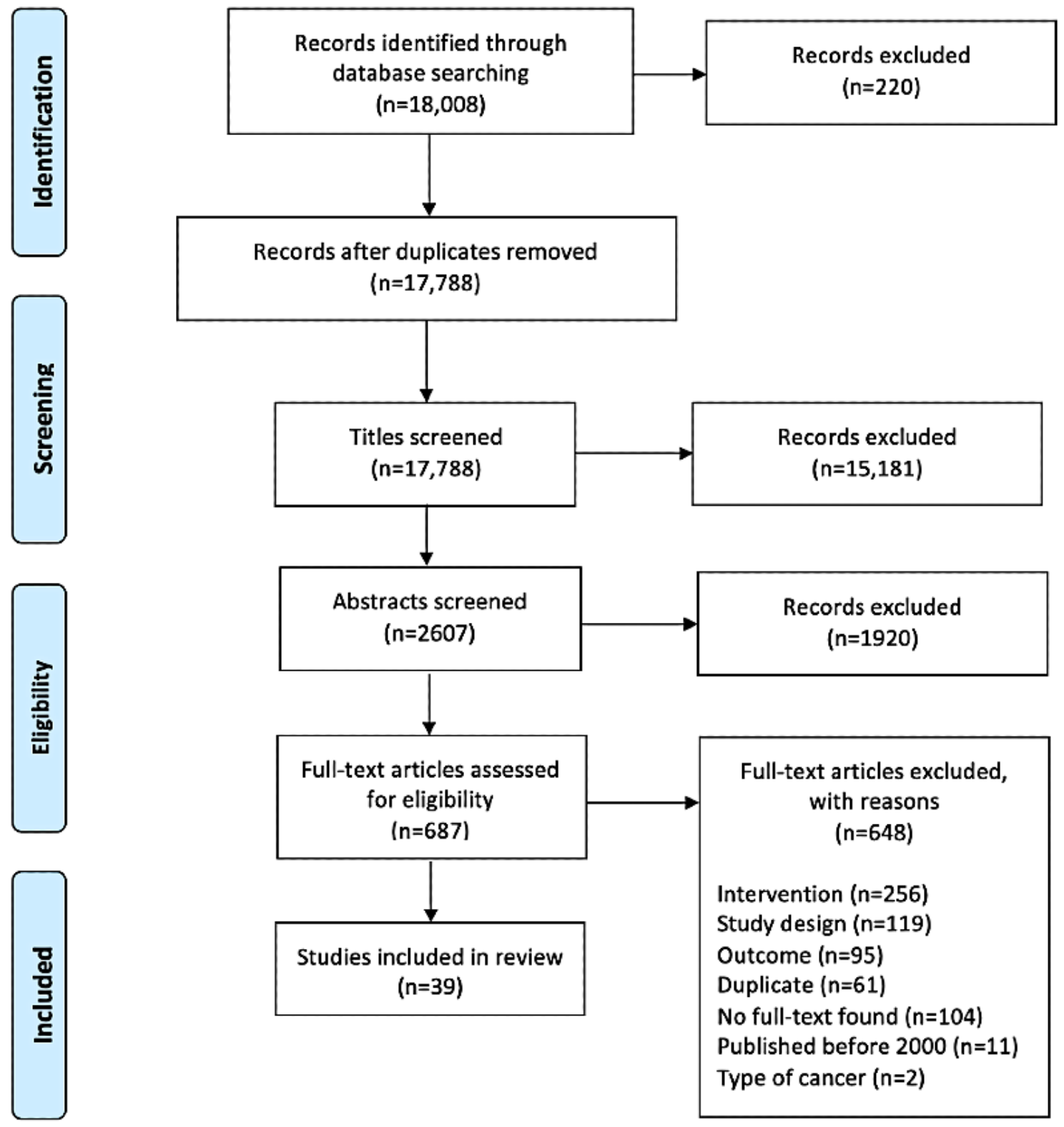


Table 1. Summary of included randomized controlled trials $(n=30)$.

\begin{tabular}{|c|c|c|c|c|c|c|c|c|}
\hline Study & Location & $\begin{array}{l}\text { Type of } \\
\text { cancer }\end{array}$ & $\begin{array}{l}\text { Intervention } \\
\text { type }\end{array}$ & $\begin{array}{l}\text { Nature of in- } \\
\text { tervention }\end{array}$ & $\begin{array}{l}\text { Total } \\
\text { sample } \\
\text { size }\end{array}$ & Population & $\begin{array}{l}\text { Summary of inter- } \\
\text { vention }\end{array}$ & Outcomes \\
\hline $\begin{array}{l}\text { Vidal et al } \\
\text { [51] }\end{array}$ & $\begin{array}{l}\text { Barcelona, } \\
\text { Spain }\end{array}$ & Breast & mHealth & Reminder & 12,786 & $\begin{array}{l}\text { Breast cancer } \\
\text { screening target } \\
\text { population of the } \\
\text { southern Barcelona } \\
\text { metropolitan area }\end{array}$ & $\begin{array}{l}\text { Text message re- } \\
\text { minder } 3 \text { days be- } \\
\text { fore a scheduled } \\
\text { appointment with } \\
\text { or without a mes- } \\
\text { sage, with a new } \\
\text { appointment date } \\
\text { if requested }\end{array}$ & $\begin{array}{l}\text { Proportion attend- } \\
\text { ing an appoint- } \\
\text { ment before Octo- } \\
\text { ber } 31,2011 \text { (3-5 } \\
\text { months after the } \\
\text { intervention) }\end{array}$ \\
\hline $\begin{array}{l}\text { Kerrison et al } \\
\text { [41] }\end{array}$ & $\begin{array}{l}\text { United King- } \\
\text { dom }\end{array}$ & Breast & mHealth & Reminder & 2240 & $\begin{array}{l}\text { Women (aged } 47- \\
53 \text { years) who } \\
\text { were due to be in- } \\
\text { vited for their first } \\
\text { routine breast } \\
\text { screen }\end{array}$ & $\begin{array}{l}\text { Text message re- } \\
\text { minder } 48 \text { hours } \\
\text { before the appoint- } \\
\text { ment and an addi- } \\
\text { tional text message } \\
\text { if they did not at- } \\
\text { tend the initial ap- } \\
\text { pointment }\end{array}$ & $\begin{array}{l}\text { Proportion attend- } \\
\text { ing the appoint- } \\
\text { ment within } 60 \\
\text { days of the initial } \\
\text { appointment }\end{array}$ \\
\hline $\begin{array}{l}\text { Rashid et al } \\
{[47]}\end{array}$ & $\begin{array}{l}\text { Klang, } \\
\text { Malaysia }\end{array}$ & Cervical & mHealth & Reminder & 1000 & $\begin{array}{l}\text { Women (aged 20- } \\
65 \text { years) residing } \\
\text { in Klang who had } \\
\text { a nonpositive } \mathrm{Pa}- \\
\text { panicolaou test in } \\
\text { the previous year } \\
\text { and were due for } \\
\text { repeat screening }\end{array}$ & $\begin{array}{l}\text { Text message re- } \\
\text { minder for a repeat } \\
\text { Papanicolaou test } \\
\text { within a month } \\
\text { from the date of re- } \\
\text { call }\end{array}$ & $\begin{array}{l}\text { Proportion com- } \\
\text { pleting the Papani- } \\
\text { colaou test within } \\
8 \text { weeks }\end{array}$ \\
\hline $\begin{array}{l}\text { Wanyoro and } \\
\text { Kabiru [52] }\end{array}$ & $\begin{array}{l}\text { Thika, } \\
\text { Kenya }\end{array}$ & Cervical & mHealth & Reminder & 286 & $\begin{array}{l}\text { Women (aged } 25- \\
70 \text { years) attending } \\
\text { the general outpa- } \\
\text { tient clinic who } \\
\text { had never had cer- } \\
\text { vical cancer } \\
\text { screening, who } \\
\text { owned a mobile } \\
\text { phone, and who } \\
\text { had normal cervi- } \\
\text { cal Papanicolaou } \\
\text { test after the initial } \\
\text { baseline screening }\end{array}$ & $\begin{array}{l}4 \text { text message re- } \\
\text { minders in a period } \\
\text { of } 2 \text { weeks }\end{array}$ & $\begin{array}{l}\text { Proportion } \\
\text { screened for cervi- } \\
\text { cal cancer at the } \\
\text { same site within } 2 \\
\text { weeks }\end{array}$ \\
\hline Huf et al [39] & $\begin{array}{l}\text { United King- } \\
\text { dom }\end{array}$ & Cervical & mHealth & Reminder & 14,587 & $\begin{array}{l}\text { Women (aged 24- } \\
64 \text { years) }\end{array}$ & $\begin{array}{l}1 \text { of } 6 \text { text message } \\
\text { reminders: a sim- } \\
\text { ple reminder, gener- } \\
\text { al practice endorse- } \\
\text { ment, total and } \\
\text { proportional social } \\
\text { norms messages, } \\
\text { and gain- and loss- } \\
\text { framed messages }\end{array}$ & $\begin{array}{l}\text { - Proportion who } \\
\text { screened within } 18 \\
\text { weeks after the re- } \\
\text { minder }\end{array}$ \\
\hline
\end{tabular}




\begin{tabular}{|c|c|c|c|c|c|c|c|c|}
\hline Study & Location & $\begin{array}{l}\text { Type of } \\
\text { cancer }\end{array}$ & $\begin{array}{l}\text { Intervention } \\
\text { type }\end{array}$ & $\begin{array}{l}\text { Nature of in- } \\
\text { tervention }\end{array}$ & $\begin{array}{l}\text { Total } \\
\text { sample } \\
\text { size }\end{array}$ & Population & $\begin{array}{l}\text { Summary of inter- } \\
\text { vention }\end{array}$ & Outcomes \\
\hline Sly et al [50] & $\begin{array}{l}\text { New York, } \\
\text { United } \\
\text { States }\end{array}$ & $\mathrm{CRC}^{\mathrm{b}}$ & mHealth & Reminder & 24 & $\begin{array}{l}\text { Adults (aged }>50 \\
\text { years) with referral } \\
\text { for screening } \\
\text { colonoscopy with } \\
\text { no personal or } \\
\text { family history of } \\
\text { CRC or any chron- } \\
\text { ic gastrointestinal } \\
\text { disorder, with tele- } \\
\text { phone service, and } \\
\text { who spoke English }\end{array}$ & $\begin{array}{l}\text { Standard naviga- } \\
\text { tion, a scheduling } \\
\text { telephone call and } \\
2 \text { text message ap- } \\
\text { pointment re- } \\
\text { minders }\end{array}$ & $\begin{array}{l}\text { - Colonoscopy com- } \\
\text { pletion within } 3 \\
\text { months }\end{array}$ \\
\hline $\begin{array}{l}\text { Hagoel et al } \\
\text { [36] }\end{array}$ & Israel & $\mathrm{CRC}$ & mHealth & Reminder & 48,091 & $\begin{array}{l}\text { Adults (aged 50-74 } \\
\text { years) with no diag- } \\
\text { nosis of an inflam- } \\
\text { matory bowel dis- } \\
\text { ease or a bowel } \\
\text { malignancy, who } \\
\text { had not undergone } \\
\text { colonoscopy with- } \\
\text { in the previous } 3 \\
\text { years, and who had } \\
\text { not performed } \\
\text { FOBT }{ }^{c} \text { in the previ- } \\
\text { ous year }\end{array}$ & $\begin{array}{l}\text { Text message re- } \\
\text { minders including } \\
\text { interrogative or } \\
\text { noninterrogative } \\
\text { messages }\end{array}$ & $\begin{array}{l}\text { Proportion com- } \\
\text { pleting FOBT at } 6 \\
\text { months }\end{array}$ \\
\hline $\begin{array}{l}\text { Coronado et al } \\
\text { [32] }\end{array}$ & $\begin{array}{l}\text { United } \\
\text { States }\end{array}$ & $\mathrm{CRC}$ & mHealth & Reminder & 2010 & $\begin{array}{l}\text { Adults (aged 50-75 } \\
\text { years) not up to } \\
\text { date with CRC } \\
\text { screening and with } \\
\text { a clinic visit in the } \\
\text { previous year }\end{array}$ & $\begin{array}{l}2 \text { text message re- } \\
\text { minders with or } \\
\text { without a live } \\
\text { phone call }\end{array}$ & - $\quad$ FIT $^{\mathrm{d}}$ kit return rate \\
\hline Hirst et al [38] & $\begin{array}{l}\text { United King- } \\
\text { dom }\end{array}$ & CRC & mHealth & Reminder & 8269 & $\begin{array}{l}\text { Adults (aged 60-74 } \\
\text { years) }\end{array}$ & $\begin{array}{l}\text { Usual care and a } \\
\text { text message re- } \\
\text { minder if they had } \\
\text { not returned their } \\
\text { test kit within } 8 \\
\text { weeks }\end{array}$ & $\begin{array}{l}\text { Proportion return- } \\
\text { ing test kit at the } \\
\text { end of an 18-week } \\
\text { screening episode }\end{array}$ \\
\hline Lam et al [61] & Hong Kong & CRC & mHealth & Reminder & 500 & $\begin{array}{l}\text { Adults (aged 40-70 } \\
\text { years) who were } \\
\text { asymptomatic and } \\
\text { had a previous } \\
\text { negative FIT test } \\
\text { and who were ex- } \\
\text { pected for an annu- } \\
\text { al FIT screening in } \\
\text { the subsequent } \\
\text { year }\end{array}$ & $\begin{array}{l}\text { A WhatsApp mes- } \\
\text { sage reminder sent } \\
1 \text { month before the } \\
\text { due date for subse- } \\
\text { quent FIT }\end{array}$ & $\begin{array}{l}\text { - Proportion success- } \\
\text { fully returning the } \\
\text { FIT kit }\end{array}$ \\
\hline $\begin{array}{l}\text { Coronado et al } \\
{[33]}\end{array}$ & $\begin{array}{l}\text { Los Angeles, } \\
\text { United } \\
\text { States }\end{array}$ & CRC & mHealth & Reminder & 1767 & $\begin{array}{l}\text { Adults (aged } 50-75 \\
\text { years) who were } \\
\text { overdue for CRC } \\
\text { screening and had } \\
\text { attended at least } \\
\text { two clinic visits } \\
\text { within the past } 24 \\
\text { months }\end{array}$ & $\begin{array}{l}\text { Text message } \\
\text { prompt before re- } \\
\text { ceipt of the FIT kit } \\
\text { with } 2 \text { automated } \\
\text { phone call re- } \\
\text { minders or with } 2 \\
\text { automated phone } \\
\text { calls and up to } 3 \\
\text { live phone call re- } \\
\text { minders }\end{array}$ & $\begin{array}{l}\text { Proportion com- } \\
\text { pleting the FIT kit } \\
\text { within } 6 \text { months }\end{array}$ \\
\hline $\begin{array}{l}\text { Hwang et al } \\
{[40]}\end{array}$ & $\begin{array}{l}\text { United } \\
\text { States }\end{array}$ & $\mathrm{CRC}$ & Social media & Peer support & 306 & & & \\
\hline
\end{tabular}




\begin{tabular}{|c|c|c|c|c|c|c|c|c|}
\hline Study & Location & $\begin{array}{l}\text { Type of } \\
\text { cancer }\end{array}$ & $\begin{array}{l}\text { Intervention } \\
\text { type }\end{array}$ & $\begin{array}{l}\text { Nature of in- } \\
\text { tervention }\end{array}$ & $\begin{array}{l}\text { Total } \\
\text { sample } \\
\text { size }\end{array}$ & Population & $\begin{array}{l}\text { Summary of inter- } \\
\text { vention }\end{array}$ & Outcomes \\
\hline & & & & & & $\begin{array}{l}\text { Adults (aged 50-75 } \\
\text { years) who had no } \\
\text { previous diagnosis } \\
\text { of CRC, had no } \\
\text { history of inflam- } \\
\text { matory bowel dis- } \\
\text { ease, and were not } \\
\text { up to date with } \\
\text { CRC screening }\end{array}$ & $\begin{array}{l}\text { Study-specific } \\
\text { web-based Spark- } \\
\text { Team to access the } \\
\text { narratives and inter- } \\
\text { act with the narra- } \\
\text { tors (positive role } \\
\text { models) and other } \\
\text { participants }\end{array}$ & $\begin{array}{l}\text { Proportion } \\
\text { screened for CRC } \\
\text { at } 6 \text { months } \\
\text { (FOBT, sigmoi- } \\
\text { doscopy, or } \\
\text { colonoscopy) }\end{array}$ \\
\hline $\begin{array}{l}\text { Lakkis et al } \\
\text { [43] }\end{array}$ & $\begin{array}{l}\text { Beirut, } \\
\text { Lebanon }\end{array}$ & Breast & mHealth & $\begin{array}{l}\text { Mixed (edu- } \\
\text { cation and } \\
\text { reminder) }\end{array}$ & 385 & $\begin{array}{l}\text { Women (aged } 40- \\
75 \text { years) who had } \\
\text { not undergone a } \\
\text { mammogram in the } \\
\text { past } 2 \text { years }\end{array}$ & $\begin{array}{l}\text { Educational and } \\
\text { general invitation } \\
\text { text message for } \\
\text { mammography and } \\
3 \text { additional text } \\
\text { reminders }\end{array}$ & $\begin{array}{l}\text { - Completion of a } \\
\text { mammography }\end{array}$ \\
\hline $\begin{array}{l}\text { Chung et al } \\
\text { [31] }\end{array}$ & $\begin{array}{l}\text { Republic of } \\
\text { Korea }\end{array}$ & Breast & mHealth & $\begin{array}{l}\text { Mixed (edu- } \\
\text { cation and } \\
\text { reminder) }\end{array}$ & 202 & $\begin{array}{l}\text { Women (aged 20- } \\
65 \text { years) who un- } \\
\text { derwent surgery } \\
\text { for breast cancer, } \\
\text { excluding those } \\
\text { with distant metas- } \\
\text { tasis or recurrent } \\
\text { breast cancer }\end{array}$ & $\begin{array}{l}\text { Usual care and } 1 \\
\text { text message re- } \\
\text { minder and } 1 \text { edu- } \\
\text { cational text mes- } \\
\text { sage }\end{array}$ & $\begin{array}{l}\text { - Adherent to } \\
\text { monthly BSE } \text { for }^{\mathrm{e}} \\
5 \text { out of } 6 \text { months }\end{array}$ \\
\hline $\begin{array}{l}\text { Heydari and } \\
\text { Noroozi [37] }\end{array}$ & $\begin{array}{l}\text { Bushehr, } \\
\text { Iran }\end{array}$ & Breast & mHealth & $\begin{array}{l}\text { Mixed (edu- } \\
\text { cation and } \\
\text { reminder) }\end{array}$ & 120 & $\begin{array}{l}\text { Women (aged } \geq 40 \\
\text { years) who were } \\
\text { elementary school } \\
\text { teachers, were not } \\
\text { pregnant or breast- } \\
\text { feeding, had no } \\
\text { history of cancer, } \\
\text { had no family histo- } \\
\text { ry of breast cancer, } \\
\text { had not had breast } \\
\text { biopsy experience } \\
\text { and mammography } \\
\text { in the past } 3 \text { years }\end{array}$ & $\begin{array}{l}\text { Multimedia educa- } \\
\text { tion session } \\
\text { through a CD and } \\
\text { text messages; } 1-2 \\
\text { educational text } \\
\text { messages sent on a } \\
\text { weekly basis for } 1 \\
\text { month and a re- } \\
\text { minder about } \\
\text { mammography }\end{array}$ & $\begin{array}{l}\text { Proportion com- } \\
\text { pleting mammogra- } \\
\text { phy } \\
\text { - } \quad \text { Intention to get a } \\
\text { mammography }\end{array}$ \\
\hline Lee et al [44] & $\begin{array}{l}\text { Minnesota, } \\
\text { United } \\
\text { States }\end{array}$ & Breast & mHealth & $\begin{array}{l}\text { Mixed (edu- } \\
\text { cation and } \\
\text { navigation) }\end{array}$ & 131 & $\begin{array}{l}\text { Korean American } \\
\text { immigrant women } \\
\text { (aged 40-79 years) } \\
\text { who had not re- } \\
\text { ceived a mammo- } \\
\text { gram in the past } 2 \\
\text { years }\end{array}$ & $\begin{array}{l}\text { mMammogram } \\
\text { mobile app deliver- } \\
\text { ing } 8 \text { - } 21 \text { messages } \\
\text { over a } 7 \text {-day period }\end{array}$ & $\begin{array}{l}\text { Proportion receiv- } \\
\text { ing mammography } \\
\text { or with a sched- } \\
\text { uled appointment } \\
\text { within } 6 \text { months } \\
\text { Intention to re- } \\
\text { ceive a mammogra- } \\
\text { phy in the future } \\
\text { on a 4-point scale } \\
\text { (1=not within a } \\
\text { year, } 2=\text { within a } \\
\text { year, 3=within } 3 \\
\text { months, and } \\
4=\text { within } 1 \text { month) }\end{array}$ \\
\hline $\begin{array}{l}\text { Khademolhos- } \\
\text { seini et al [42] }\end{array}$ & $\begin{array}{l}\text { Bushehr, } \\
\text { Iran }\end{array}$ & Cervical & mHealth & $\begin{array}{l}\text { Mixed (edu- } \\
\text { cation and } \\
\text { reminder) }\end{array}$ & 95 & & $\begin{array}{l}\text { Educational train- } \\
\text { ing through text } \\
\text { messaging, elec- } \\
\text { tronic posters, info- } \\
\text { graphics, podcasts, } \\
\text { and video tutorial } \\
\text { and a reminder to } \\
\text { perform a Papanico- } \\
\text { laou smear test }\end{array}$ & $\begin{array}{l}\text { - Completion of the } \\
\text { Papanicolaou test } \\
\text { within } 3 \text { months }\end{array}$ \\
\hline
\end{tabular}




\begin{tabular}{|c|c|c|c|c|c|c|c|c|}
\hline Study & Location & $\begin{array}{l}\text { Type of } \\
\text { cancer }\end{array}$ & $\begin{array}{l}\text { Intervention } \\
\text { type }\end{array}$ & $\begin{array}{l}\text { Nature of in- } \\
\text { tervention }\end{array}$ & $\begin{array}{l}\text { Total } \\
\text { sample } \\
\text { size }\end{array}$ & Population & $\begin{array}{l}\text { Summary of inter- } \\
\text { vention }\end{array}$ & Outcomes \\
\hline & & & & & & $\begin{array}{l}\text { Women who were } \\
\text { able to read and } \\
\text { write, were mar- } \\
\text { ried for at least } 6 \\
\text { months, had a } \\
\text { smartphone, had } \\
\text { no history of geni- } \\
\text { tal tract cancer in } \\
\text { their family, and } \\
\text { had no experience } \\
\text { of doing a Papani- } \\
\text { colaou smear test } \\
\text { in the past } 3 \text { years }\end{array}$ & & \\
\hline $\begin{array}{l}\text { Richman et al } \\
\text { [49] }\end{array}$ & $\begin{array}{l}\text { North Caroli- } \\
\text { na, United } \\
\text { States }\end{array}$ & $\begin{array}{l}\text { Cervical } \\
\text { or rectal }\end{array}$ & mHealth & $\begin{array}{l}\text { Mixed (edu- } \\
\text { cation and } \\
\text { reminder) }\end{array}$ & 264 & $\begin{array}{l}\text { Adults (aged } 18-26 \\
\text { years) who attend- } \\
\text { ed the university } \\
\text { and who were vol- } \\
\text { untarily initiating } \\
\text { the first HPV } \text { vac- }^{\text {f }} \text { acine dose from the } \\
\text { cine campus student } \\
\text { health center }\end{array}$ & $\begin{array}{l}7 \text { electronic email } \\
\text { or text messages } \\
\text { once per month for } \\
7 \text { months }\end{array}$ & $\begin{array}{l}\text { Proportion com- } \\
\text { pleting HPV dose } \\
3 \text { vaccine }\end{array}$ \\
\hline $\begin{array}{l}\text { Adler et al } \\
\text { [62] }\end{array}$ & $\begin{array}{l}\text { United } \\
\text { States }\end{array}$ & Cervical & mHealth & $\begin{array}{l}\text { Mixed (edu- } \\
\text { cation and } \\
\text { reminder) }\end{array}$ & 95 & $\begin{array}{l}\text { Women (aged } 21- \\
65 \text { years) with no } \\
\text { past hysterectomy } \\
\text { with cervical re- } \\
\text { moval or known } \\
\text { HIV infection }\end{array}$ & $\begin{array}{l}\text { Referral and } 3 \text { text } \\
\text { messages delivered } \\
\text { at } 30 \text {-day intervals } \\
\text { over a period of } 90 \\
\text { days after enroll- } \\
\text { ment }\end{array}$ & $\begin{array}{l}\text { Proportion who } \\
\text { underwent cervi- } \\
\text { cal cancer screen- } \\
\text { ing } 150 \text { days after } \\
\text { enrollment }\end{array}$ \\
\hline $\begin{array}{l}\text { Erwin et al } \\
{[34]}\end{array}$ & $\begin{array}{l}\text { Kilimanjaro } \\
\text { and Arusha } \\
\text { regions, Tan- } \\
\text { zania }\end{array}$ & Cervical & mHealth & $\begin{array}{l}\text { Mixed (edu- } \\
\text { cation and } \\
\text { reminder) }\end{array}$ & 851 & $\begin{array}{l}\text { Women (aged 25- } \\
49 \text { years) with ac- } \\
\text { cess to a mobile } \\
\text { phone living in the } \\
\text { catchment areas of } \\
\text { Mawenzi Regional } \\
\text { Referral Hospital } \\
\text { and Meru District } \\
\text { Hospital }\end{array}$ & $\begin{array}{l}15 \text { unique text } \\
\text { messages delivered } \\
\text { over } 21 \text { days with } \\
\text { or without a trans- } \\
\text { portation e-vouch- } \\
\text { er covering return } \\
\text { transportation to } \\
\text { the nearest screen- } \\
\text { ing clinic }\end{array}$ & $\begin{array}{l}\text { - } \quad \text { Proportion attend- } \\
\text { ing cervical cancer } \\
\text { screening within } \\
60 \text { days }\end{array}$ \\
\hline $\begin{array}{l}\text { Firmino- } \\
\text { Machado et al } \\
{[35]}\end{array}$ & Portugal & Cervical & mHealth & $\begin{array}{l}\text { Mixed (edu- } \\
\text { cation and } \\
\text { reminder) }\end{array}$ & 1220 & $\begin{array}{l}\text { Women (aged 25- } \\
49 \text { years) eligible } \\
\text { for screening and } \\
\text { registered at prima- } \\
\text { ry health care units } \\
\text { that perform sys- } \\
\text { tematic written let- } \\
\text { ter invitations for } \\
\text { screening }\end{array}$ & $\begin{array}{l}\text { Automated or cus- } \\
\text { tomized text mes- } \\
\text { sages and phone } \\
\text { calls, followed by } \\
\text { text message re- } \\
\text { minders of the ap- } \\
\text { pointment (step 1), } \\
\text { phone calls by } \\
\text { clinical secretaries } \\
\text { (step 2), and phone } \\
\text { calls or face-to- } \\
\text { face interviews by } \\
\text { doctors (step 3) }\end{array}$ & $\begin{array}{l}\text { Proportion adher- } \\
\text { ent to cervical } \\
\text { cancer screening } \\
\text { at } 45 \text { (step 1), } 90 \\
\text { (step } 1+2 \text { ), and } \\
150 \text { days after the } \\
\text { initial invitation } \\
\text { (step } 1+2+3 \text { ) }\end{array}$ \\
\hline $\begin{array}{l}\text { Linde et al } \\
\text { [65] }\end{array}$ & Tanzania & Cervical & mHealth & $\begin{array}{l}\text { Mixed (edu- } \\
\text { cation and } \\
\text { reminder) }\end{array}$ & 689 & $\begin{array}{l}\text { Women (aged 25- } \\
60 \text { years) who had } \\
\text { tested positive for } \\
\text { HPV during a pa- } \\
\text { tient-initiated op- } \\
\text { portunistic screen- } \\
\text { ing } 14 \text { months ear- } \\
\text { lier }\end{array}$ & $\begin{array}{l}10 \text { educative text } \\
\text { messages ( } 1 \text { per } \\
\text { month) and } 5 \text { re- } \\
\text { minders }(14,7, \text { and } \\
1 \text { day before the } \\
\text { scheduled screen- } \\
\text { ing appointment) } \\
\text { over a } 10 \text {-month } \\
\text { period }\end{array}$ & $\begin{array}{l}\text { - Proportion attend- } \\
\text { ing the scheduled } \\
\text { screening appoint- } \\
\text { ment within } 30 \\
\text { days }\end{array}$ \\
\hline $\begin{array}{l}\text { Romli et al } \\
\text { [63] }\end{array}$ & $\begin{array}{l}\text { Kedah, } \\
\text { Malaysia }\end{array}$ & Cervical & mHealth & & 210 & & & \\
\hline
\end{tabular}




\begin{tabular}{|c|c|c|c|c|c|c|c|c|}
\hline Study & Location & $\begin{array}{l}\text { Type of } \\
\text { cancer }\end{array}$ & $\begin{array}{l}\text { Intervention } \\
\text { type }\end{array}$ & $\begin{array}{l}\text { Nature of in- } \\
\text { tervention }\end{array}$ & $\begin{array}{l}\text { Total } \\
\text { sample } \\
\text { size }\end{array}$ & Population & $\begin{array}{l}\text { Summary of inter- } \\
\text { vention }\end{array}$ & Outcomes \\
\hline & & & & $\begin{array}{l}\text { Mixed (edu- } \\
\text { cation and } \\
\text { reminder) }\end{array}$ & & $\begin{array}{l}\text { Women en- } \\
\text { trepreneurs (aged } \\
\text { 20-65 years) who } \\
\text { received financial } \\
\text { help from Amanah } \\
\text { Ikhtiar Malaysia } \\
\text { and who were or } \\
\text { had been previous- } \\
\text { ly married }\end{array}$ & $\begin{array}{l}\text { A 30-minute educa- } \\
\text { tional talk, a 5- } \\
\text { minute video on } \\
\text { Papanicolaou } \\
\text { smear test proce- } \\
\text { dures, experience } \\
\text { sharing from a cer- } \\
\text { vical cancer sur- } \\
\text { vivor, distribution } \\
\text { of pamphlet on } \\
\text { cervical cancer and } \\
\text { Papanicolaou } \\
\text { smear testing, and } \\
2 \text { text message re- } \\
\text { minders sent over } \\
\text { a 3-month period }\end{array}$ & $\begin{array}{l}\text { Proportion having } \\
\text { a Papanicolaou } \\
\text { smear test }\end{array}$ \\
\hline $\begin{array}{l}\text { Baker et al } \\
{[30]}\end{array}$ & $\begin{array}{l}\text { Chicago, } \\
\text { United } \\
\text { States }\end{array}$ & $\mathrm{CRC}$ & mHealth & $\begin{array}{l}\text { Mixed (edu- } \\
\text { cation, re- } \\
\text { minder, and } \\
\text { navigation) }\end{array}$ & 450 & $\begin{array}{l}\text { Adults (aged 51-75 } \\
\text { years) with pre- } \\
\text { ferred language } \\
\text { listed as English or } \\
\text { Spanish and with a } \\
\text { negative FOBT }\end{array}$ & $\begin{array}{l}\text { A mailed reminder } \\
\text { letter and FIT kit } \\
\text { with postage-paid } \\
\text { envelope, automat- } \\
\text { ed telephone and } \\
\text { text message re- } \\
\text { minders, and per- } \\
\text { sonal telephone } \\
\text { outreach by a } \\
\text { screening naviga- } \\
\text { tor after } 3 \text { months }\end{array}$ & $\begin{array}{l}\text { Proportion com- } \\
\text { pleting either } \\
\text { FOBT or } \\
\text { colonoscopy with- } \\
\text { in } 6 \text { months of the } \\
\text { date the patient } \\
\text { was due for annual } \\
\text { screening }\end{array}$ \\
\hline $\begin{array}{l}\text { Muller et al } \\
{[46]}\end{array}$ & $\begin{array}{l}\text { Anchorage, } \\
\text { Alaska }\end{array}$ & CRC & mHealth & $\begin{array}{l}\text { Mixed (edu- } \\
\text { cation and } \\
\text { reminder) }\end{array}$ & 2386 & $\begin{array}{l}\text { Alaska Native or } \\
\text { American Indian } \\
\text { adults (aged 40-75 } \\
\text { years) with no his- } \\
\text { tory of CRC or } \\
\text { colectomy enrolled } \\
\text { with the Southcen- } \\
\text { tral Foundation } \\
\text { health care system } \\
\text { and eligible for } \\
\text { screening }\end{array}$ & $\begin{array}{l}\text { A maximum of } 3 \\
\text { text messages over } \\
2 \text { months }\end{array}$ & $\begin{array}{l}\text { Proportion } \\
\text { screened (FIT, } \\
\text { FOBT, flexible } \\
\text { sigmoidoscopy, or } \\
\text { colonoscopy) }\end{array}$ \\
\hline $\begin{array}{l}\text { Miller et al } \\
{[45]}\end{array}$ & $\begin{array}{l}\text { North Caroli- } \\
\text { na, United } \\
\text { States }\end{array}$ & $\mathrm{CRC}$ & mHealth & $\begin{array}{l}\text { Mixed (edu- } \\
\text { cation and } \\
\text { decision aid) }\end{array}$ & 450 & $\begin{array}{l}\text { English-speaking } \\
\text { adults (aged 50-74 } \\
\text { years) who were } \\
\text { scheduled to see a } \\
\text { primary care } \\
\text { provider and were } \\
\text { due for CRC } \\
\text { screening }\end{array}$ & $\begin{array}{l}\text { mPATH-CRC, an } \\
\text { iPad app providing } \\
\text { screening informa- } \\
\text { tion, help with } \\
\text { screening decision, } \\
\text { self-ordering a } \\
\text { screening test, and } \\
\text { automated electron- } \\
\text { ic messages to } \\
\text { complete the cho- } \\
\text { sen test }\end{array}$ & $\begin{array}{l}\text { - CRC screening } \\
\text { completed within } \\
24 \text { weeks } \\
\text { - Intention to re- } \\
\text { ceive screening } \\
\text { within the next } 6 \\
\text { months }\end{array}$ \\
\hline $\begin{array}{l}\text { Reiter et al } \\
{[48]}\end{array}$ & $\begin{array}{l}\text { United } \\
\text { States }\end{array}$ & Rectal & mHealth & $\begin{array}{l}\text { Mixed (edu- } \\
\text { cation and } \\
\text { reminder) }\end{array}$ & 150 & $\begin{array}{l}\text { Gay or bisexual } \\
\text { men (aged } 18-25 \\
\text { years) residing in } \\
\text { the United States } \\
\text { who had not re- } \\
\text { ceived any HPV } \\
\text { vaccine doses }\end{array}$ & $\begin{array}{l}\text { Population-target- } \\
\text { ed, individually } \\
\text { tailored content } \\
\text { about HPV and } \\
\text { monthly HPV vac- } \\
\text { cination reminders } \\
\text { sent via email } \\
\text { and/or text mes- } \\
\text { sage }\end{array}$ & $\begin{array}{l}\text { Proportion com- } \\
\text { pleting all } 3 \text { doses } \\
\text { of the HPV vac- } \\
\text { cine }\end{array}$ \\
\hline $\begin{array}{l}\text { Wong et al } \\
\text { [53] }\end{array}$ & Hong Kong & CRC & mHealth & $\begin{array}{l}\text { Mixed (edu- } \\
\text { cation and } \\
\text { reminder) }\end{array}$ & 629 & & & \\
\hline
\end{tabular}




\begin{tabular}{|c|c|c|c|c|c|c|c|c|}
\hline Study & Location & $\begin{array}{l}\text { Type of } \\
\text { cancer }\end{array}$ & $\begin{array}{l}\text { Intervention } \\
\text { type }\end{array}$ & $\begin{array}{l}\text { Nature of in- } \\
\text { tervention }\end{array}$ & $\begin{array}{l}\text { Total } \\
\text { sample } \\
\text { size }\end{array}$ & Population & $\begin{array}{l}\text { Summary of inter- } \\
\text { vention }\end{array}$ & Outcomes \\
\hline & & & & & & $\begin{array}{l}\text { Adults (aged } 40-70 \\
\text { years) at average } \\
\text { risk of CRC who } \\
\text { had a negative FIT } \\
\text { result in their first } \\
\text { screening round for } \\
\text { the study }\end{array}$ & $\begin{array}{l}\text { Generic text mes- } \\
\text { sage about the im- } \\
\text { portance of regular } \\
\text { CRC screening and } \\
\text { the time and venue } \\
\text { of FIT tube re- } \\
\text { trieval }\end{array}$ & $\begin{array}{l}\text { Proportion success- } \\
\text { fully returning } \\
\text { completed FIT } \\
\text { specimen within } 6 \\
\text { months }\end{array}$ \\
\hline $\begin{array}{l}\text { Mahmud et al } \\
\text { [64] }\end{array}$ & $\begin{array}{l}\text { United } \\
\text { States }\end{array}$ & $\mathrm{CRC}$ & mHealth & $\begin{array}{l}\text { Mixed (edu- } \\
\text { cation and } \\
\text { reminder) }\end{array}$ & 71 & $\begin{array}{l}\text { Adults (aged 18-75 } \\
\text { years) scheduled } \\
\text { for outpatient } \\
\text { colonoscopy with- } \\
\text { in } 2 \text { months of ini- } \\
\text { tial contact }\end{array}$ & $\begin{array}{l}9 \text { text messages } \\
\text { sent in the week } \\
\text { before the sched- } \\
\text { uled procedure }\end{array}$ & $\begin{array}{l}\text { Proportion who at- } \\
\text { tended their } \\
\text { scheduled appoint- } \\
\text { ment }\end{array}$ \\
\hline
\end{tabular}

\footnotetext{
${ }^{\mathrm{a}} \mathrm{mHealth}$ : mobile health.

${ }^{\mathrm{b}} \mathrm{CRC}$ : colorectal cancer.

${ }^{\mathrm{c}}$ FOBT: fecal occult blood test.

${ }^{\mathrm{d}}$ FIT: fecal immunochemical test.

${ }^{\mathrm{e}} \mathrm{BSE}$. breast self-exam.

${ }^{\mathrm{f}} \mathrm{HPV}$ : human papilloma virus.
}

The most common reminder strategies used were text message reminders [29-39,41-43,46-55,57-65]. Educational strategies most commonly included general health information about the specific cancer and information about cancer screening, including the importance of screening. Although text messages were commonly used to deliver educational information [34,35,37,42-44,46,48,49,53-55,59,62,64,65], some studies also used electronic posters or infographics, CDs, videos, mobile apps, and podcasts $[37,42,44,45,55,59,63]$. Education was also provided through in-person educational or training sessions in some cases in addition to a social media or mHealth strategy or in the comparison groups [55,63]. Educational interventions using social media included social media campaigns [56] or sharing information or daily posts about screening or cancer with participants who were members of a group (virtual community) on a social media platform $[66,67]$. Peer support interventions on social media also leveraged groups to support participants of that virtual community through the sharing of personal stories and narratives [40]. Outcomes were measured at several time points, including the proportion attending a scheduled appointment or those participating in screening within 2 weeks [52], a month [65], 45 days [35], 60 days [29,34,41,47], 3 months [35,42,50], 3-5 months [38,39,51], or 6 months $[30,31,33,36,40,45,53]$.

There was wide variability in the study participants. For example, the included participants were targeted based on geographical region in some studies $[34,51,56]$ or by their profession as elementary school teachers [37], entrepreneurs [63], or university students [49,59]. Some studies were targeted to specific racial and cultural groups $[44,46,54,58,67]$, whereas others included gay and bisexual men only [48] or women who were HIV positive [60]. The intervention intensity also differed between the studies. For example, some interventions included sending only a single text message reminder [29,31,33,38,39,41,51], whereas others included sending 22 text messages over 16 days [54] or 21 messages over a 7-day period [44]. For social media interventions, participants in one study received three daily posts over a 12-week period [67] or as many as 20 posts per day over 5 days [66]. 
Table 2. Summary of included pre- and postintervention studies $(n=9)$.

\begin{tabular}{|c|c|c|c|c|c|c|c|c|}
\hline Study & Location & $\begin{array}{l}\text { Type of } \\
\text { cancer }\end{array}$ & $\begin{array}{l}\text { Intervention } \\
\text { type }\end{array}$ & $\begin{array}{l}\text { Nature of in- } \\
\text { tervention }\end{array}$ & $\begin{array}{l}\text { Total sam- } \\
\text { ple size }\end{array}$ & Population & $\begin{array}{l}\text { Summary of inter- } \\
\text { vention }\end{array}$ & Outcomes \\
\hline $\begin{array}{l}\text { Ganta et } \\
\text { al [60] }\end{array}$ & $\begin{array}{l}\text { Nevada, United } \\
\text { States }\end{array}$ & Cervical & mHealth $^{\mathrm{a}}$ & Reminder & 473 & $\begin{array}{l}\text { HIV-infected } \\
\text { women (aged } \\
\geq 18 \text { years) at } \\
\text { the HIV Well- } \\
\text { ness Center }\end{array}$ & $\begin{array}{l}\text { Reminders to } \\
\text { schedule a Papan- } \\
\text { icolaou test via } 3 \\
\text { sequential text } \\
\text { messages and } \\
\text { subsequently by } \\
3 \text { phone call at- } \\
\text { tempts }\end{array}$ & $\begin{array}{l}\text { Proportion complet- } \\
\text { ing the Papanico- } \\
\text { laou test }\end{array}$ \\
\hline $\begin{array}{l}\text { Lee et al } \\
{[58]}\end{array}$ & $\begin{array}{l}\text { Minnesota, } \\
\text { United States }\end{array}$ & Cervical & mHealth & $\begin{array}{l}\text { Education or } \\
\text { awareness }\end{array}$ & 30 & $\begin{array}{l}\text { Korean Ameri- } \\
\text { can women } \\
\text { (aged 21-29 } \\
\text { years) with no } \\
\text { previous receipt } \\
\text { of a Papanico- } \\
\text { laou test with } \\
\text { up-to-date } \\
\text { health insurance }\end{array}$ & $\begin{array}{l}\text { 7-day text mes- } \\
\text { sage-based inter- } \\
\text { vention including } \\
\text { quizzes and ques- } \\
\text { tions and engage- } \\
\text { ment in conversa- } \\
\text { tion }\end{array}$ & $\begin{array}{l}\text { Proportion receiving } \\
\text { a Papanicolaou test } \\
\text { within } 3 \text { months } \\
\text { Intent to receive a } \\
\text { Papanicolaou test } \\
\text { within a year }\end{array}$ \\
\hline $\begin{array}{l}\text { Lemos et } \\
\text { al [59] }\end{array}$ & $\begin{array}{l}\text { Madeira, Portu- } \\
\text { gal }\end{array}$ & Cervical & mHealth & $\begin{array}{l}\text { Education or } \\
\text { awareness }\end{array}$ & 144 & $\begin{array}{l}\text { Female college } \\
\text { students recruit- } \\
\text { ed from various } \\
\text { undergraduate } \\
\text { courses of } \\
\text { Madeira Univer- } \\
\text { sity }\end{array}$ & $\begin{array}{l}5 \text { structured text } \\
\text { messages deliv- } \\
\text { ered over } 5 \\
\text { weeks and an edu- } \\
\text { cational video in- } \\
\text { tervention lasting } \\
12 \text { minutes }\end{array}$ & $\begin{array}{l}\text { Intention to get a } \\
\text { Papanicolaou test } \\
\text { measured on a 5- } \\
\text { point Likert scale } \\
\text { from } 1 \text { (definitely } \\
\text { will not do) to } 5 \\
\text { (definitely will do) }\end{array}$ \\
\hline $\begin{array}{l}\text { Le and } \\
\text { Holt [54] }\end{array}$ & United States & Cervical & mHealth & $\begin{array}{l}\text { Education or } \\
\text { awareness }\end{array}$ & 52 & $\begin{array}{l}\text { Church-attend- } \\
\text { ing African- } \\
\text { American wom- } \\
\text { en (aged 21-65 } \\
\text { years) with no } \\
\text { previous medi- } \\
\text { cal history of } \\
\text { cervical cancer } \\
\text { or hysterectomy }\end{array}$ & $\begin{array}{l}22 \text { text messages } \\
\text { delivered over } 16 \\
\text { days, containing } \\
\text { health-specific } \\
\text { and spiritually } \\
\text { based content }\end{array}$ & $\begin{array}{l}\text { - Intent to get a Papan- } \\
\text { icolaou smear test in } \\
\text { the next } 6 \text { months }\end{array}$ \\
\hline $\begin{array}{l}\text { Lyson et } \\
\text { al [66] }\end{array}$ & United States & Cervical & Social media & $\begin{array}{l}\text { Education or } \\
\text { awareness }\end{array}$ & 782 & $\begin{array}{l}\text { Women (aged } \\
\geq 18 \text { years) who } \\
\text { lived in the } \\
\text { United States, } \\
\text { spoke English } \\
\text { as their primary } \\
\text { language, and } \\
\text { did not have } \\
\text { cervical cancer }\end{array}$ & $\begin{array}{l}\text { Health Connect } \\
\text { web-based plat- } \\
\text { form where partic- } \\
\text { ipants were as- } \\
\text { signed to groups } \\
\text { of } 9 \text { and where } \\
\text { each participant } \\
\text { was randomly } \\
\text { distributed a set } \\
\text { of } 20 \text { tweets or } \\
\text { messages per day } \\
\text { over } 5 \text { days in a } \\
\text { personalized } \\
\text { message feed }\end{array}$ & $\begin{array}{l}\text { - Proportion ever had } \\
\text { a Papanicolaou test } \\
\text { Proportion ever re- } \\
\text { ceived the HPV } \\
\text { vaccine }\end{array}$ \\
\hline $\begin{array}{l}\text { Key et al } \\
{[67]}\end{array}$ & $\begin{array}{l}\text { Kentucky, Unit- } \\
\text { ed States }\end{array}$ & $\mathrm{CRC}^{\mathrm{c}}$ & Social media & $\begin{array}{l}\text { Education or } \\
\text { awareness }\end{array}$ & 60 & $\begin{array}{l}\text { Appalachian } \\
\text { Kentuckians } \\
\text { (aged } \geq 50 \\
\text { years) noncom- } \\
\text { pliant with cur- } \\
\text { rent screening } \\
\text { guidelines }\end{array}$ & $\begin{array}{l}\text { Participants } \\
\text { joined a closed } \\
\text { Facebook group } \\
\text { and were present- } \\
\text { ed with } 3 \text { daily } \\
\text { Facebook posts } \\
\text { during the 12- } \\
\text { week intervention }\end{array}$ & $\begin{array}{l}\text { Proportion ever re- } \\
\text { ceived a } \\
\text { colonoscopy or } \\
\text { FOBT }^{\text {d }}\end{array}$ \\
\hline
\end{tabular}




\begin{tabular}{|c|c|c|c|c|c|c|c|c|}
\hline Study & Location & $\begin{array}{l}\text { Type of } \\
\text { cancer }\end{array}$ & $\begin{array}{l}\text { Intervention } \\
\text { type }\end{array}$ & $\begin{array}{l}\text { Nature of in- } \\
\text { tervention }\end{array}$ & $\begin{array}{l}\text { Total sam- } \\
\text { ple size }\end{array}$ & Population & $\begin{array}{l}\text { Summary of inter- } \\
\text { vention }\end{array}$ & Outcomes \\
\hline $\begin{array}{l}\text { Jessup et } \\
\text { al [56] }\end{array}$ & $\begin{array}{l}\text { Massachusetts, } \\
\text { United States }\end{array}$ & Lung & Social media & $\begin{array}{l}\text { Education or } \\
\text { awareness }\end{array}$ & $\begin{array}{l}\text { Variable } \\
\text { depending } \\
\text { on plat- } \\
\text { form }\end{array}$ & $\begin{array}{l}\text { Patients, care- } \\
\text { givers, and } \\
\text { health care } \\
\text { providers with- } \\
\text { in a } 60 \text {-mile ra- } \\
\text { dius of a large } \\
\text { quaternary med- } \\
\text { ical center and } \\
2 \text { affiliated off- } \\
\text { campus imag- } \\
\text { ing sites. Pa- } \\
\text { tient campaign } \\
\text { targeted current } \\
\text { and former } \\
\text { smokers (aged } \\
\geq 55 \text { years), fe- } \\
\text { males (aged } \\
\geq 55 \text { years), pa- } \\
\text { tients and em- } \\
\text { ployees of the } \\
\text { academic medi- } \\
\text { cal center (aged } \\
\geq 18 \text { years), and } \\
\text { caregivers } \\
\text { (aged } \geq 18 \\
\text { years) }\end{array}$ & $\begin{array}{l}\text { Patient awareness } \\
\text { campaign on } \\
\text { Facebook and } \\
\text { Google and } \\
\text { provider cam- } \\
\text { paign on } \\
\text { LinkedIn and } \\
\text { Twitter }\end{array}$ & $\begin{array}{l}\text { Number of LDCT } \\
\text { examinations per } \\
\text { week before and af- } \\
\text { ter the campaign }\end{array}$ \\
\hline $\begin{array}{l}\text { Fornos et } \\
\text { al [57] }\end{array}$ & $\begin{array}{l}\text { Texas, United } \\
\text { States }\end{array}$ & Cervical & mHealth & $\begin{array}{l}\text { Mixed (edu- } \\
\text { cation, re- } \\
\text { minders, and } \\
\text { navigation) }\end{array}$ & 32,807 & $\begin{array}{l}\text { Women (aged } \\
\geq 18 \text { years) en- } \\
\text { rolled in Care- } \\
\text { Link who were } \\
\text { not up to date } \\
\text { with Papanico- } \\
\text { laou screening } \\
\text { or actively ob- } \\
\text { taining Papani- } \\
\text { colaou test ap- } \\
\text { pointments }\end{array}$ & $\begin{array}{l}\text { Newsletters, pub- } \\
\text { lic service an- } \\
\text { nouncements, au- } \\
\text { tomated client re- } \\
\text { minders includ- } \\
\text { ing text mes- } \\
\text { sages, and com- } \\
\text { munity outreach }\end{array}$ & $\begin{array}{l}\text { 3-year cervical can- } \\
\text { cer screening rate }\end{array}$ \\
\hline $\begin{array}{l}\text { Capik } \\
\text { and } \\
\text { Gozum } \\
{[55]}\end{array}$ & $\begin{array}{l}\text { Erzurum, } \\
\text { Turkey }\end{array}$ & Prostate & mHealth & $\begin{array}{l}\text { Mixed (edu- } \\
\text { cation and } \\
\text { reminders) }\end{array}$ & 75 & $\begin{array}{l}\text { Men (aged } 41- \\
65 \text { years) work- } \\
\text { ing in } 2 \text { public } \\
\text { institutions who } \\
\text { had not re- } \\
\text { ceived a } \\
\text { prostate cancer } \\
\text { diagnosis }\end{array}$ & $\begin{array}{l}\text { Poster announce- } \\
\text { ments, interactive } \\
\text { educational ses- } \\
\text { sion, access to } \\
\text { website, desk cal- } \\
\text { endar information } \\
\text { and reminders, } \\
\text { monthly email re- } \\
\text { minders, flyers, } \\
\text { and } 1 \text { text mes- } \\
\text { sage }\end{array}$ & $\begin{array}{l}\text { Proportion having } \\
\text { had a PSA } \mathrm{PA}^{\mathrm{f}} \text { test in } \\
\text { the last } 3 \text { months } \\
\text { Proportion having } \\
\text { had a prostate exam- } \\
\text { ination in the last } 3 \\
\text { months }\end{array}$ \\
\hline
\end{tabular}

${ }^{\mathrm{a}}$ mHealth: mobile health.

${ }^{\mathrm{b}} \mathrm{HPV}$ : human papilloma virus.

${ }^{\mathrm{c}} \mathrm{CRC}$ : colorectal cancer.

${ }^{\mathrm{d}}$ FOBT: fecal occult blood test.

${ }^{\mathrm{e}}$ LDCT: low-dose computed tomography.

${ }^{f}$ PSA: prostate-specific antigen.

\section{Quality Assessment}

Risk of bias assessments for the included studies are shown in Figures 2 and 3. Briefly, 27\% (8/30) of the included RCTs were classified as high risk, 23\% (7/30) as having some concerns, and the remainder $(15 / 30,50 \%)$ were classified as low risk. Common reasons for being classified as high risk included having some concerns in several domains, including bias arising from the randomization process, effect of assignment to intervention, and measurement of the outcome. All pre- and postintervention studies were classified as high risk. Figure 4 displays the funnel plot used to check for publication bias. The $\mathrm{x}$-axis represents the effect estimates, whereas the $\mathrm{y}$-axis represents the study size or precision. The funnel plot generated 
may suggest some publication bias because of the lack of studies small effect sizes and variances. in the bottom left corner of the plot representing studies with

Figure 2. Risk of bias assessment for the included randomized controlled trials $(n=30)$ created using the Robvis tool.

\begin{tabular}{|c|c|c|c|c|c|c|}
\hline & \multicolumn{6}{|c|}{ Risk of bias domains } \\
\hline & $\mathrm{D} 1$ & D2 & D3 & $\mathrm{D} 4$ & D5 & Overall \\
\hline Adler et al [62] & - & $\odot$ & & - & 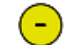 & (x) \\
\hline Arcas et al [29] & + & - & $x$ & + & - & \\
\hline Baker et al [30] & $\oplus$ & $\oplus$ & $\oplus$ & $\oplus$ & $\oplus$ & $\oplus$ \\
\hline Chung et al [31] & $\oplus$ & $\odot$ & ( & $\Theta$ & $\oplus$ & $\bigodot$ \\
\hline Coronado et al [32] & $\oplus$ & $\oplus$ & $\oplus$ & $\oplus$ & $\oplus$ & $\oplus$ \\
\hline Coronado et al [33] & $\oplus$ & $\oplus$ & $\oplus$ & $\oplus$ & $\oplus$ & $\oplus$ \\
\hline Erwin et al [34] & $\oplus$ & $\oplus$ & $\oplus$ & $\oplus$ & $\oplus$ & $\oplus$ \\
\hline Firmino-Machado et al [35] & $\Theta$ & $\oplus$ & $\oplus$ & $\oplus$ & $\oplus$ & $\bigodot$ \\
\hline Hagoel et al [36] & $\oplus$ & $\oplus$ & $\oplus$ & $\oplus$ & $\oplus$ & $\oplus$ \\
\hline Heydari and Noroozi [37] & - & $\odot$ & $\oplus$ & - & $\oplus$ & (X) \\
\hline Hirst et al [38] & $\oplus$ & $\oplus$ & $\oplus$ & $\oplus$ & $\oplus$ & $\oplus$ \\
\hline Huf et al [39] & ๑ & $\oplus$ & $\oplus$ & $\oplus$ & $\oplus$ & $\oplus$ \\
\hline Hwang et al [40] & $\Theta$ & $\oplus$ & $\oplus$ & $\oplus$ & $\oplus$ & $\Theta$ \\
\hline Kerrison et al [41] & † & $\oplus$ & $\oplus$ & $\oplus$ & $\oplus$ & $\oplus$ \\
\hline Khademolhosseini et al [42] & $\odot$ & $\bigodot$ & $\oplus$ & $\odot$ & $\oplus$ & ) \\
\hline Lakkis et al [43] & $\oplus$ & $\bigodot$ & $\oplus$ & $\bigodot$ & $\oplus$ & $\bigodot$ \\
\hline Lam et al [61] & $\odot$ & $\oplus$ & $\oplus$ & $\oplus$ & $\oplus$ & $\odot$ \\
\hline Lee et al [44] & $\oplus$ & $\oplus$ & $\oplus$ & $\odot$ & $\oplus$ & $\odot$ \\
\hline Linde et al [65] & 8 & $\mathrm{x}$ & 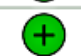 & $\oplus$ & $\odot$ & 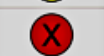 \\
\hline Mahmoud et al 64] & - & $\oplus$ & $\Theta$ & $\oplus$ & $\oplus$ & $\Theta$ \\
\hline Miller et al [45] & $\oplus$ & $\oplus$ & $\oplus$ & $\oplus$ & $\oplus$ & $\oplus$ \\
\hline Muller et al [46] & $\oplus$ & $\oplus$ & $\oplus$ & $\oplus$ & $\oplus$ & $\oplus$ \\
\hline Rashid et al [47] & † & $\oplus$ & $\oplus$ & $\oplus$ & $\oplus$ & $\oplus$ \\
\hline Reiter et al [48] & $\oplus$ & $\oplus$ & $\oplus$ & $\oplus$ & $\oplus$ & $\oplus$ \\
\hline Richman et al [49] & † & $\oplus$ & $\oplus$ & $\oplus$ & $\oplus$ & $\oplus$ \\
\hline Romli et al [63] & $\mathrm{X}$ & $\odot$ & $\oplus$ & $\odot$ & $\oplus$ & \\
\hline Sly et al [50] & - & $\odot$ & $\oplus$ & $\oplus$ & $\oplus$ & \\
\hline Vidal et al [51] & $\odot$ & $\oplus$ & $\oplus$ & $\oplus$ & $\odot$ & Q \\
\hline Wanyoro and Kabiru [52] & $\oplus$ & $\oplus$ & $\oplus$ & $\oplus$ & $\oplus$ & $\oplus$ \\
\hline Wong et al [53] & $\oplus$ & $\oplus$ & $\oplus$ & ( & $\oplus$ & $\oplus$ \\
\hline & 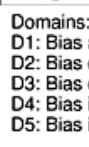 & & & & & e concerns \\
\hline
\end{tabular}


Figure 3. Risk of bias assessment for the included pre- and postintervention studies $(n=9)$.

\begin{tabular}{|c|c|c|c|c|c|c|c|c|}
\hline Study & D1 & D2 & D3 & D4 & D5 & D6 & D7 & Overall* \\
\hline $\begin{array}{l}\text { Capik and } \\
\text { Gozum [55] }\end{array}$ & & & & & & & & \\
\hline $\begin{array}{l}\text { Fornos et al } \\
{[57]}\end{array}$ & & & & & & & & \\
\hline $\begin{array}{l}\text { Ganta et al } \\
{[60]}\end{array}$ & & & & & & & & \\
\hline $\begin{array}{l}\text { Jessup et al } \\
{[56]}\end{array}$ & & & & & & & & \\
\hline Key et al [67 & & & & & & & & \\
\hline $\begin{array}{l}\text { Le and Holt } \\
{[54]}\end{array}$ & & & & & & & & \\
\hline Lee et al [ 58 & & & & & & & & \\
\hline $\begin{array}{l}\text { Lemos et al } \\
{[59]}\end{array}$ & & & & & & & & \\
\hline $\begin{array}{l}\text { Lyson et al } \\
{[66]}\end{array}$ & & & & & & & & \\
\hline
\end{tabular}

*Overall risk of bias judgement was assigned low risk if the study was judged to be at low risk for all individual domains; medium/unclear risk if the study was judged to be at medium/unclear risk in at least one domain, but not at high risk of bias for any domain; and high risk of bias if the study was judged to bet at high risk in at least one domain or at medium/unclear risk in multiple domains in a way that substantially lowers confidence in the result.

\section{Domains:}

D1: Intervention independent of other changes

D2: Shape of intervention effect pre-specified

D3: Intervention unlikely to affect data collection

D4: Knowledge of the allocated interventions adequately prevented during the study

D5: Incomplete outcome data

D6: Selective outcome reporting

D7: Other risk of bias

\section{Judgement:}

High risk

Medium/Unclear risk

Low risk 
Figure 4. Funnel plot of publication bias for the randomized controlled trials reporting on the primary outcome. OR: odds ratio.

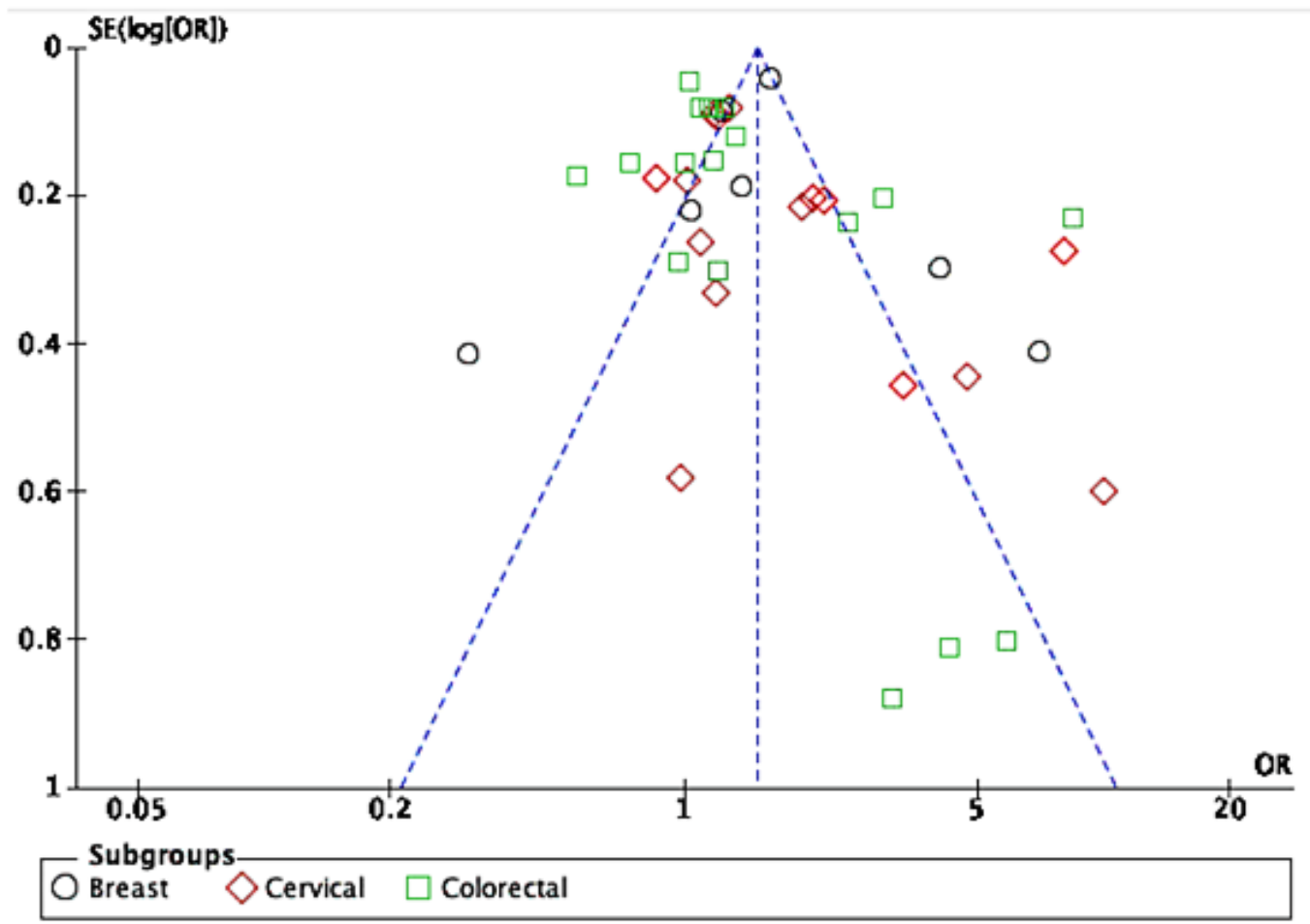

\section{Primary and Secondary Outcomes}

The absolute effect of being screened in the intervention arms was $22.22 \%(13,115 / 59,017)$. There was an absolute risk difference of $14 \%$ (95\% CI 13.12-14.33) between the intervention and comparison arms, with the proportion screened in the comparison arms being $35.94 \%$ (12,524/34,872). When stratified by cancer type, the absolute proportion screened in the intervention arms was $71.68 \%(3935 / 5489)$ for breast cancer compared with $64.11 \%(7096 / 11,067)$ in the comparison arms (risk difference 8\%; 95\% CI 6.08-9.06). For cervical cancer, there were $35.23 \%(2382 / 6760)$ screened in the intervention arms compared with $28.26 \%$ (1548/5478) in the comparison arms. For CRC, the proportion screened in the intervention arms was $14.53 \%(6798 / 46,768)$ and $21.17 \%(3880 / 18,327)$ in the comparison arms, with a risk difference of $6 \%(95 \% \mathrm{CI}$ 5.96-7.31).

The overall pooled OR for cancer screening participation among the included RCTs was 1.49 (95\% CI 1.31-1.70; Figure 5), indicating that the odds of getting screened increased by $49 \%$ for those who received a social media or mHealth intervention. However, considerable heterogeneity was observed $\left(\mathrm{I}^{2}=88 \%\right)$. Similar effect estimates were observed when stratified by cancer type, with the largest effect observed for cervical cancer screening studies (OR 1.71, 95\% CI 1.34-2.19; Figure 5). Stratification by cancer type did not reduce the heterogeneity. When we conducted a sensitivity analysis excluding trials assessed to have a high risk of bias, the overall pooled OR and $\mathrm{I}^{2}$ remained stable (OR 1.54, 95\% CI 1.33-1.78; Figure 6). The overall pooled OR was not significant when including only studies measuring screening participation through self-reporting (OR 2.09, 95\% CI 0.96-4.53). The overall pooled effect estimate remained stable when including only studies that captured the outcome through administrative records (OR 1.46, 95\% CI 1.28-1.66). When we included only studies conducted in LMIC settings $(n=3)$, the overall pooled OR was $3.29(95 \%$ CI $1.02-10.60)$ with considerable heterogeneity $\left(\mathrm{I}^{2}=93 \%\right)$. However, the pooled OR increased to 5.50 (95\% CI 3.19-9.51) with only moderate heterogeneity $\left(\mathrm{I}^{2}=38 \%\right)$ when only studies with a low risk of bias were included $(n=2)$. We also conducted subgroup analyses by meta-analyzing studies based on the nature of the intervention. The results showed an overall pooled effect estimate of 1.23 (95\% CI 1.08-1.41) for reminder interventions (Figure 7) and 2.07 (95\% CI 1.49-5.83) for mixed interventions (Figure 8). Heterogeneity did not change when subgroup analyses were conducted. 
Figure 5. Forest plot for the randomized controlled trials reporting on the primary outcome of cancer screening participation categorized by type of cancer $(\mathrm{n}=30)$.

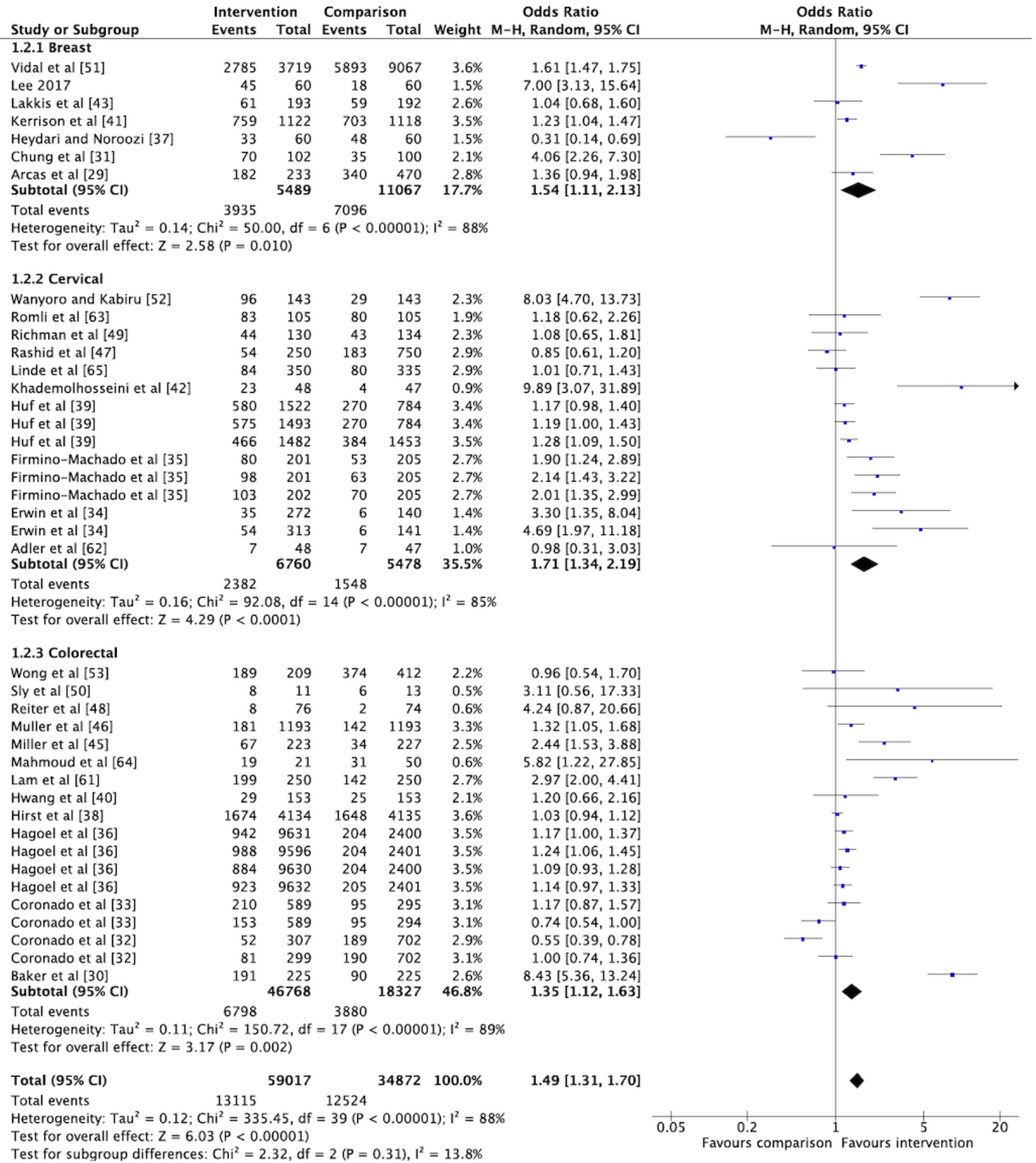


Figure 6. Sensitivity analysis for the primary outcome of interest of cancer screening participation without inclusion of randomized controlled trials with a high risk of bias $(n=22)$.

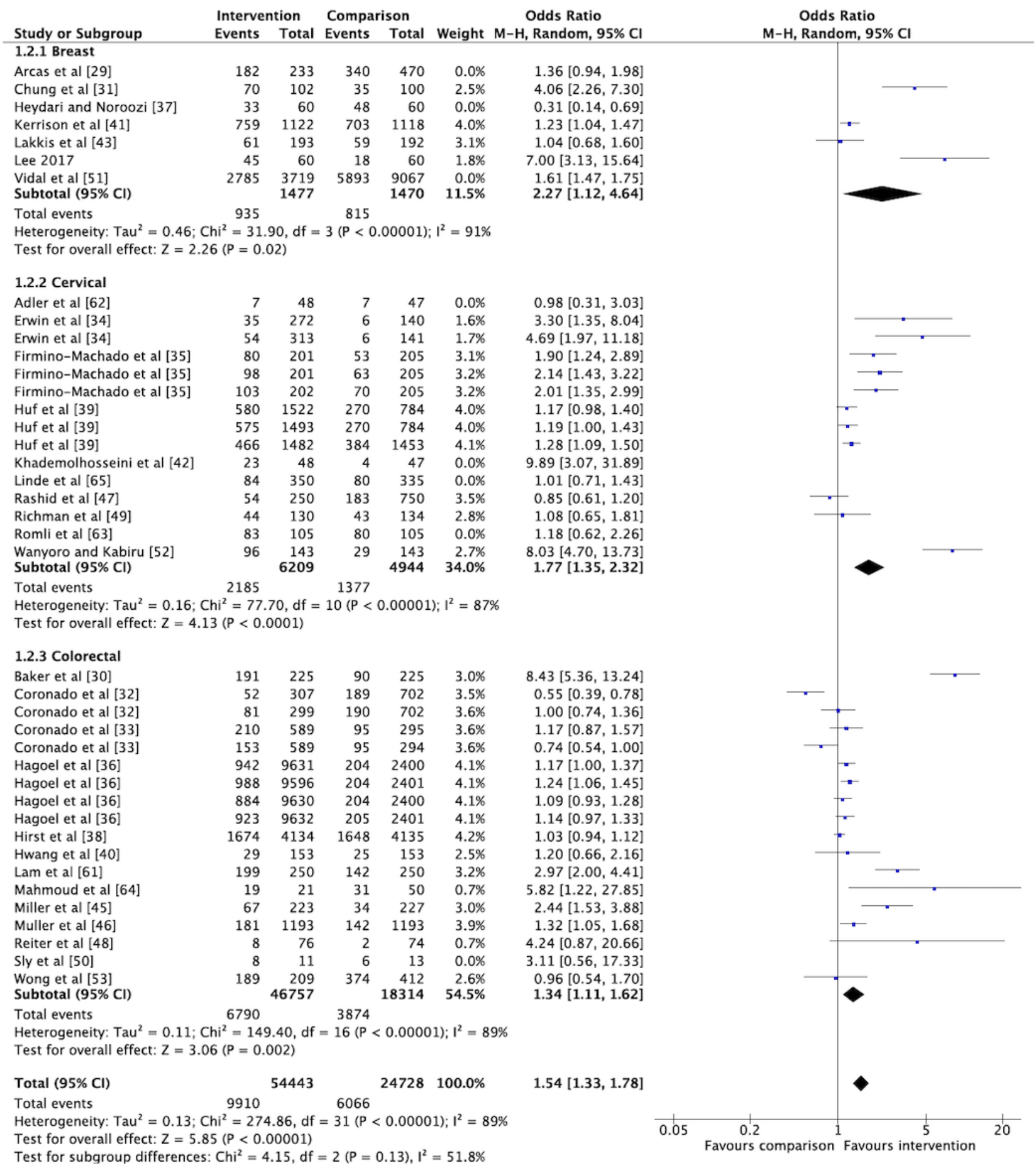


Figure 7. Forest plot for the reminder interventions reporting on the primary outcome of cancer screening participation $(n=12)$.

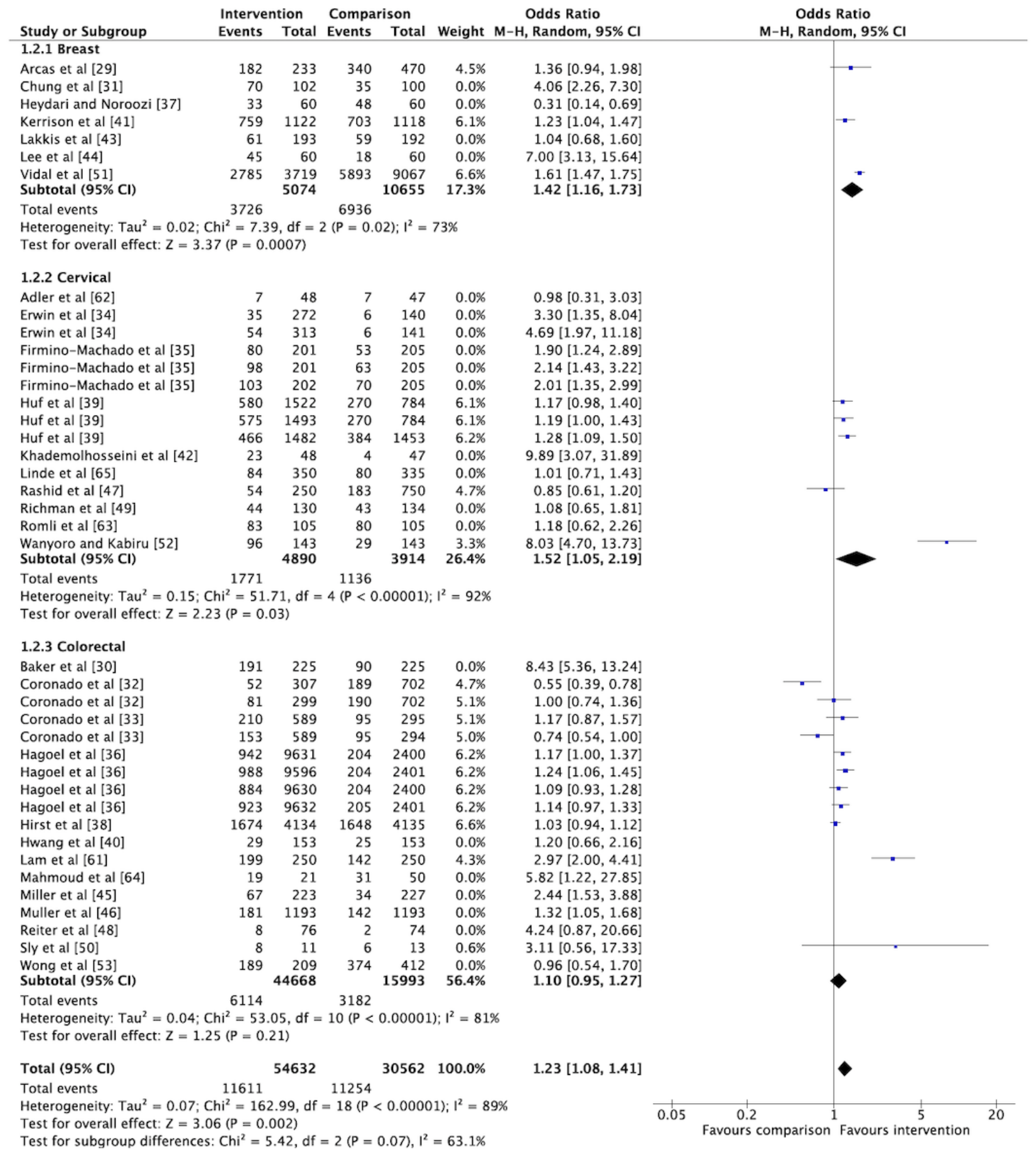


Figure 8. Forest plot for the mixed interventions reporting on the primary outcome of cancer screening participation $(\mathrm{n}=17)$.

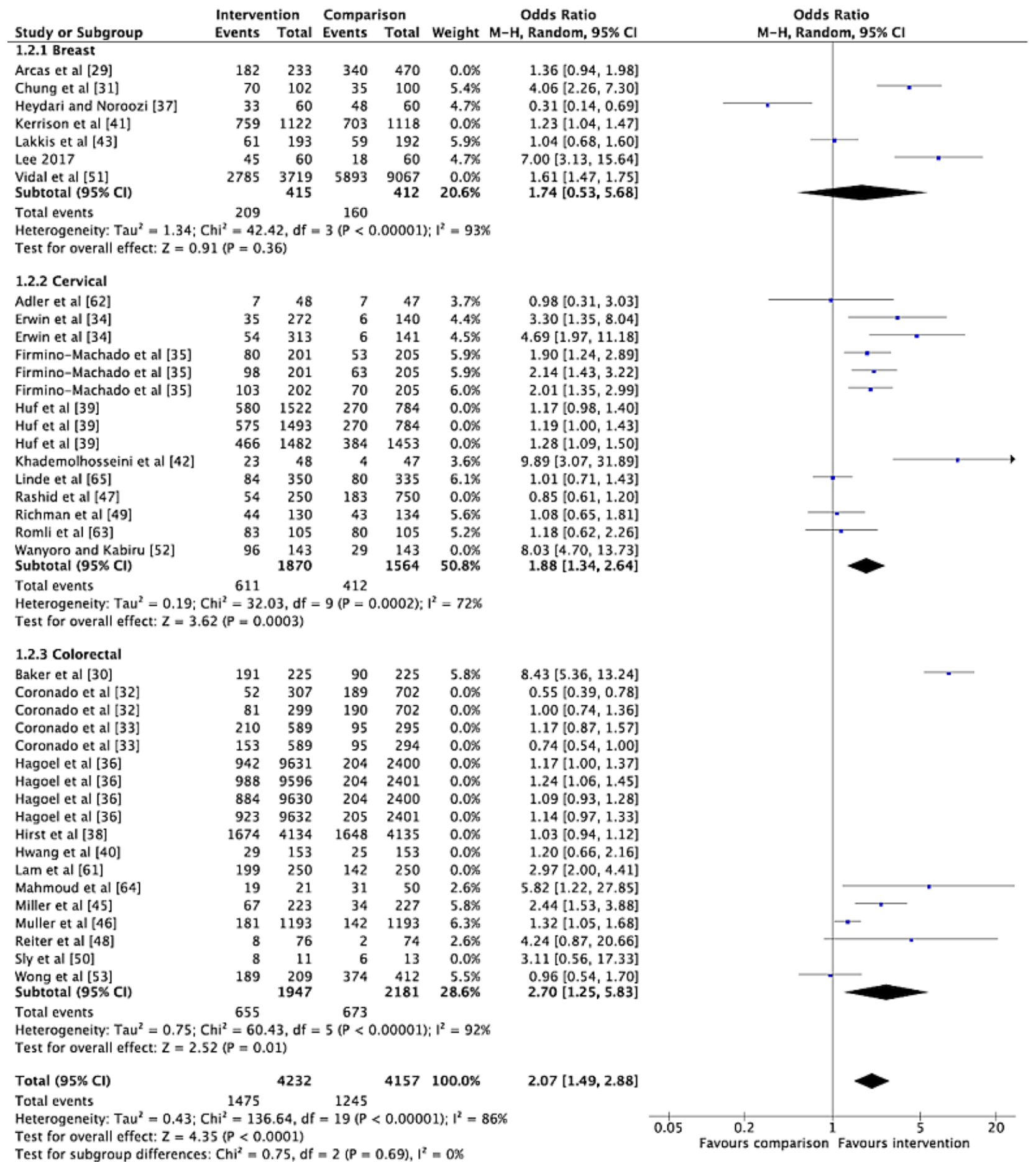

Table 3 presents the results of the secondary outcomes of screening intention. Six studies ( 3 RCTs and 3 pre- and postintervention studies) reported on screening intention, with two studies reporting on screening intention only. There was minor variability in the measurement of screening intention among the studies. For example, screening intention was treated as a dichotomous variable in some studies $[37,45,54,58]$ or scored using a four-point [44] or five-point [59] Likert scale in others. Half of the studies $(3 / 6,50 \%)$ focused on cervical cancer, followed by breast cancer $(2 / 6,33 \%)$ and CRC $(1 / 6,17 \%)$. The intention to screen increased in all studies reporting on this

outcome, except for one in which it decreased. The highest increase in screening intention was observed in the study by Lee et al [58], where there was a $24 \%$ absolute increase in the intent to receive a Papanicolaou test postintervention (19/30, $63 \%$ preintervention and $26 / 30,87 \%$ postintervention). The study included a 7-day text message-based intervention that included a high level of engagement with participants through quizzes, questions, and engagement in conversation [58]. Owing to the variability in how screening intention was measured or captured, we did not perform a meta-analysis on these data. 
Table 3. Cancer screening intention outcome among included studies $(n=6)$.

\begin{tabular}{|c|c|c|c|c|c|}
\hline Study & Study design & Outcome definition & $\begin{array}{l}\text { Timeframe for as- } \\
\text { sessing outcome }\end{array}$ & $\begin{array}{l}\text { Outcome in compari- } \\
\text { son group (if } \mathrm{RCT}^{\mathrm{a}} \text { ) } \\
\text { or preintervention }\end{array}$ & $\begin{array}{l}\text { Outcome in interven- } \\
\text { tion group (if RCT) or } \\
\text { postintervention }\end{array}$ \\
\hline $\begin{array}{l}\text { Heydari and } \\
\text { Noroozi [37] }\end{array}$ & RCT & $\begin{array}{l}\text { Intention to get a mammogram } \\
\text { (yes or no) }\end{array}$ & 3 months & $93 \%(56 / 60)$ & $83 \%(50 / 60)$ \\
\hline Lee et al [44] & RCT & $\begin{array}{l}\text { Intention to receive a mammo- } \\
\text { gram in the future on a 4-point } \\
\text { scale (1=not within a year, } \\
2=\text { within a year, } 3=\text { =within } 3 \\
\text { months, and } 4=\text { within } 1 \text { month) } \\
\text { among intervention and control } \\
\text { groups }\end{array}$ & $\begin{array}{l}\text { 1-week postinterven- } \\
\text { tion }\end{array}$ & $\begin{array}{l}\text { Group differences } \\
\text { preintervention }-0.64\end{array}$ & $\begin{array}{l}\text { Group differences } \\
\text { postintervention } 3.48\end{array}$ \\
\hline $\begin{array}{l}\text { Miller et al } \\
{[45]}\end{array}$ & RCT & $\begin{array}{l}\text { Intention to receive screening } \\
\text { measured through the postpro- } \\
\text { gram iPad survey }\end{array}$ & 6 months & $49 \%(112 / 227)$ & $62 \%(138 / 223)$ \\
\hline $\begin{array}{l}\text { Le and Holt } \\
{[54]}\end{array}$ & Pre- and postintervention & $\begin{array}{l}\text { Intent to get a Papanicolaou } \\
\text { smear test (yes or no) }\end{array}$ & 6 months & $48 \%(22 / 46)$ & $52 \%(24 / 46)$ \\
\hline Lee et al [58] & Pre- and postintervention & $\begin{array}{l}\text { Intent to receive a Papanicolaou } \\
\text { test (yes or no) }\end{array}$ & Within 1 year & $63 \%(19 / 30)$ & $87 \%(26 / 30)$ \\
\hline $\begin{array}{l}\text { Lemos et al } \\
\text { [59] }\end{array}$ & Pre- and postintervention & $\begin{array}{l}\text { Intention to get a Papanicolaou } \\
\text { test measured on a 5-point Lik- } \\
\text { ert scale from } 1 \text { (definitely will } \\
\text { not do) to } 5 \text { (definitely will do) }\end{array}$ & 6 weeks & $4.50(\mathrm{SD} 0.64)$ & $4.82(\mathrm{SD} 0.48)$ \\
\hline
\end{tabular}

${ }^{\mathrm{a}} \mathrm{RCT}$ : randomized controlled trial.

\section{Discussion}

\section{Principal Findings}

Our systematic review identified 39 studies describing the effectiveness of social media and mHealth interventions on cancer screening participation and/or intention. The overall pooled OR for cancer screening participation was significant, favoring the intervention arm (OR 1.49, 95\% CI 1.31-1.70). Effect sizes were similar across all cancer types, and estimates remained stable when trials deemed to be at high risk of bias were excluded, indicating that social media, and particularly mHealth interventions, can be effective for increasing cancer screening participation.

Two systematic reviews on this topic were published in 2017 $[17,18]$. Uy et al [17] evaluated the effectiveness of text messaging interventions on cancer screening and identified nine studies that met the inclusion criteria. Absolute screening rates for text messaging interventions were $1 \%-15 \%$ higher and relative screening rates were $4 \%-63 \%$ higher for intervention recipients in their study [17]. The authors concluded that text messaging interventions moderately increased screening rates for breast and cervical cancer; however, additional research is needed to better quantify this relationship [17]. Tamuzi et al [18] explored mHealth interventions for cervical cancer screening only. Their review identified 17 studies, and the authors were able to perform a meta-analysis on the results by type of intervention [18]. However, their definition of mHealth was different from ours. In their study, Tamuzi et al [18] included telephone, letter, and text message reminders, whereas only text message reminders were included in our study based on our adopted definition of mHealth interventions. Text message reminders are different from these other approaches because they are sent only to mobile devices compared with telephone calls, which may be made to landlines, for which coverage has been decreasing. In addition, text messages can be sent instantly, whereas letter or postcard reminders need to be delivered by the post. Moreover, text messages have the opportunity to reach those with no fixed addresses. For example, a recent systematic review on technology use among homeless adults showed that a majority (94\%) owned a cell phone [68]. Overall, Tamuzi et al [18] found that call reminders were the only intervention to show a statistically significant pooled effect estimate. Only one study included in their review reported on the effect of text message reminders, and a meta-analysis of this type of intervention was, therefore, not possible [18].

The results of this study enhance our understanding of the effectiveness of social media and mHealth interventions for cancer screening. Although both previous reviews were published in 2017, nearly 44\% (17/39) of the studies in this area have been published since that time. Our review provides a comprehensive and more contemporary understanding of this topic. In addition, although previous reviews focused primarily on breast and cervical cancer, our study provides valuable insights into the effectiveness of these interventions in CRC screening as well. We included 13 studies focused on CRC in our meta-analysis and found a significant pooled effect estimate, suggesting that the use of these types of interventions can be extended to CRC as well. In comparison with the study by Uy et al [17], we found that absolute screening rates between the intervention and comparison groups were higher in our study. This may suggest that multicomponent interventions that couple social media or mHealth with additional strategies may be more 
effective at increasing screening rates compared with mHealth or social media strategies alone.

The results of our study must also be understood within the larger context of interventions for cancer screening. Brouwers et al [69] conducted a systematic review of interventions for increasing cancer screening rates and looked at client reminders, client incentives, mass media, small media, group education, one-on-one education, reducing structural barriers, reducing out-of-pocket costs to clients, provider assessment and feedback, and provider incentives. Similarly, the authors found wide heterogeneity across studies and interventions and chose not to meta-analyze their data. For example, their results showed that small media interventions, including videos or printed materials such as letters, brochures, newspapers, magazines, and billboards, resulted in a point percentage increase for cancer screening participation ranging from $-32.8 \%$ to $26 \%$ among studies on breast cancer, cervical cancer, and CRC [69]. Our review showed that the absolute difference between the intervention and comparison arms was $14 \%$. The magnitude of effect varied considerably among and between intervention categories in the review by Brouwers et al [69], suggesting that additional evidence is needed for interventions related to client reminders, mass media, group education, one-on-one education, reduction of structural barriers and out-of-pocket costs, and provider incentive interventions. Given the need for additional, high-quality evidence, it is difficult to ascertain whether social media and mHealth interventions fare similar, better, or worse than non-mHealth or non-social media interventions. In addition, costs should also be considered when making any comparisons between the effectiveness of these interventions to inform the translation of these findings into practice.

Although the pooled effect estimate in our meta-analysis was consistent in the subgroup and sensitivity analyses, significant heterogeneity remained. This may be because of the variability in populations, interventions, or outcome measurement across studies. For example, the populations randomized in the studies in our review included all adults up to 79 years [44], or highly specialized populations such as emergency department patients [62] or HIV-positive individuals [60]. Moreover, many of the studies included insured samples, which may not be reflective of population-level interventions, and therefore, must be considered in the generalizability of these results. In addition, the follow-up and the intensity of each intervention varied across studies. For example, some studies may have sent a single text message reminder [37], whereas other interventions included sending multiple text messages in combination with telephone reminders [33]. Interestingly, when we looked at studies conducted in LMIC settings and excluded those with a high risk of bias, the overall pooled OR was even larger with only moderate heterogeneity. These results suggest that the effectiveness of these interventions for cancer screening participation may be more pronounced in these settings. This may be because there may be a limited number of other campaigns in these resource-low settings, whereas access to mobile phones and the internet has been reported to be comparable with that of developed nations [1].

Only a limited number of studies $(n=4)$ tested social media interventions. As such, our results are more indicative of the effectiveness of mHealth interventions. A narrative systematic review focusing on describing the characteristics of social media interventions used for cancer prevention and management found that cancer screening participation or intention was not measured in any of the 18 studies included in the review [70]. The most common outcome measured in these studies was knowledge [70]. Although research related to social media and cancer screening participation has started to emerge [71], the inclusion of this work was limited in our review, as there are few RCTs and before and after comparisons also capturing the outcome of screening participation or intention. This suggests areas for future research to generate more evidence on the use of social media interventions for cancer screening participation. In addition, very few studies have been conducted on prostate and lung cancer screening, which is similar to what was observed in a previous study [17].

Our review and meta-analysis included a variety of mHealth and social media interventions and multicomponent interventions. Our review is comprehensive and contemporary and uses a rigorous systematic approach to screen and review the literature. As such, it includes a large number of studies for the most established screening programs for breast cancer, cervical cancer, and CRC. Owing to the large number of studies included in our review, we were able to calculate pooled effect estimates by cancer type to inform practice and future research. However, this study has limitations. Although we made every effort to obtain full-text articles, there were some records identified from our search that we could not locate. We also did not calculate a Cohen $\kappa$ coefficient to report the interrater reliability between the 2 reviewers. Our review is also limited in regard to social media interventions, as only four studies were identified, with only one RCT included in the meta-analysis. This may be a reflection of current practice or due to the fact that it may be more difficult to link direct patient outcomes with the use of social media.

\section{Conclusions}

In conclusion, our results suggest that mHealth interventions may have a significant effect on cancer screening participation, particularly for breast cancer, cervical cancer, and CRC screening. Screening programs should consider the use of mHealth interventions to increase screening participation. Further research focusing on social media interventions for cancer screening participation is needed, as there was insufficient evidence available at the time of this review.

\section{Acknowledgments}

This study was supported by the Canadian Institutes of Health Research (grants FDN-148470 and GSO-157853). The funding agency had no role in the design or conduct of the study. The authors would like to acknowledge the contributions of Amina 
Benmessaoud and Alawia Sherif for their help with data management and Dr Rahim Moineddin, a thesis committee member for AR's doctoral dissertation.

\section{Conflicts of Interest}

None declared.

\section{References}

1. World Health Organization. Global Difusion of eHealth: Making Universal Health Coverage Achievable. Report of the Third Global Survey on eHealth. Geneva: World Health Organization; 2016:1-160.

2. Moorhead SA, Hazlett DE, Harrison L, Carroll JK, Irwin A, Hoving C. A new dimension of health care: systematic review of the uses, benefits, and limitations of social media for health communication. J Med Internet Res 2013;15(4):e85 [FREE Full text] [doi: 10.2196/jmir.1933] [Medline: 23615206]

3. Maher CA, Lewis LK, Ferrar K, Marshall S, De Bourdeaudhuij I, Vandelanotte C. Are health behavior change interventions that use online social networks effective? A systematic review. J Med Internet Res 2014;16(2):e40 [FREE Full text] [doi: 10.2196/jmir.2952] [Medline: 24550083]

4. Korda H, Itani Z. Harnessing social media for health promotion and behavior change. Health Promot Pract 2013 Jan;14(1):15-23. [doi: 10.1177/1524839911405850] [Medline: 21558472]

5. Bull SS, Levine DK, Black SR, Schmiege SJ, Santelli J. Social media-delivered sexual health intervention: a cluster randomized controlled trial. Am J Prev Med 2012 Nov;43(5):467-474 [FREE Full text] [doi: 10.1016/j.amepre.2012.07.022] [Medline: 23079168]

6. Prochaska JJ, Coughlin SS, Lyons EJ. Social media and mobile technology for cancer prevention and treatment. Am Soc Clin Oncol Educ Book 2017;37:128-137 [FREE Full text] [doi: 10.1200/EDBK 173841] [Medline: 28561647]

7. Hagg E, Dahinten VS, Currie LM. The emerging use of social media for health-related purposes in low and middle-income countries: a scoping review. Int J Med Inform 2018 Jul;115:92-105. [doi: 10.1016/j.ijmedinf.2018.04.010] [Medline: 29779724]

8. Ferlay J, Ervik M, Lam F, Colombet M, Mery L, Pineros M. Global cancer observatory: cancer tomorrow. International Agency for Research on Cancer. 2018. URL: https://gco.iarc.fr/tomorrow [accessed 2020-09-15]

9. Hewitson P, Glasziou P, Watson E, Towler B, Irwig L. Cochrane systematic review of colorectal cancer screening using the fecal occult blood test (hemoccult): an update. Am J Gastroenterol 2008 Jun;103(6):1541-1549. [doi: 10.1111/j.1572-0241.2008.01875.x] [Medline: $\underline{18479499]}$

10. Nelson HD, Tyne K, Naik A, Bougatsos C, Chan BK, Humphrey L, U.S. Preventive Services Task Force. Screening for breast cancer: an update for the U.S. Preventive Services Task Force. Ann Intern Med 2009 Nov 17;151(10):727-737 [FREE Full text] [doi: 10.7326/0003-4819-151-10-200911170-00009] [Medline: 19920273]

11. Peirson L, Fitzpatrick-Lewis D, Ciliska D, Warren R. Screening for cervical cancer: a systematic review and meta-analysis. Syst Rev 2013 May 24;2:35 [FREE Full text] [doi: 10.1186/2046-4053-2-35] [Medline: 23706117]

12. Sadate A, Occean BV, Beregi J, Hamard A, Addala T, de Forges H, et al. Systematic review and meta-analysis on the impact of lung cancer screening by low-dose computed tomography. Eur J Cancer 2020 Jul;134:107-114. [doi: 10.1016/j.ejca.2020.04.035] [Medline: 32502939]

13. Schreuders EH, Ruco A, Rabeneck L, Schoen RE, Sung JJ, Young GP, et al. Colorectal cancer screening: a global overview of existing programmes. Gut 2015 Oct;64(10):1637-1649. [doi: 10.1136/gutjnl-2014-309086] [Medline: 26041752]

14. Cancer Screening in Canada: An overview of screening participation for breast, cervical, and colorectal cancer. Toronto: Canadian Partnership Against Cancer. 2015. URL: https://s22457.pcdn.co/wp-content/uploads/2019/01/ Breast-Cervical-Colorectal-Screening-Participate-2015-EN.pdf [accessed 2020-09-15]

15. Youlden DR, Cramb SM, Dunn NA, Muller JM, Pyke CM, Baade PD. The descriptive epidemiology of female breast cancer: an international comparison of screening, incidence, survival and mortality. Cancer Epidemiol 2012 Jun;36(3):237-248. [doi: 10.1016/j.canep.2012.02.007] [Medline: 22459198]

16. Singh H, Bernstein CN, Samadder JN, Ahmed R. Screening rates for colorectal cancer in Canada: a cross-sectional study. CMAJ Open 2015;3(2):149-157 [FREE Full text] [doi: 10.9778/cmajo.20140073] [Medline: 26389092]

17. Uy C, Lopez J, Trinh-Shevrin C, Kwon SC, Sherman SE, Liang PS. Text messaging interventions on cancer screening rates: a systematic review. J Med Internet Res 2017 Aug 24;19(8):e296 [FREE Full text] [doi: 10.2196/jmir.7893] [Medline: 28838885]

18. Tamuzi JL. Effectiveness of mHealth to increase cervical cancer screening: systematic review of interventions. Int J Pul Res Sci 2017 Oct 27;2(3):555586. [doi: 10.19080/ijoprs.2017.02.555586]

19. Parackal M, Parackal S, Eusebius S, Mather D. The use of Facebook advertising for communicating public health messages: a campaign against drinking during pregnancy in New Zealand. JMIR Public Health Surveill 2017 Aug 10;3(3):e49 [FREE Full text] [doi: 10.2196/publichealth.7032] [Medline: 28798011] 
20. Cavallo DN, Chou WS, McQueen A, Ramirez A, Riley WT. Cancer prevention and control interventions using social media: user-generated approaches. Cancer Epidemiol Biomarkers Prev 2014 Sep;23(9):1953-1956. [doi: 10.1158/1055-9965.EPI-14-0593] [Medline: 25103820]

21. Valle CG, Tate DF. Engagement of young adult cancer survivors within a Facebook-based physical activity intervention. Transl Behav Med 2017 Dec;7(4):667-679 [FREE Full text] [doi: 10.1007/s13142-017-0483-3] [Medline: 28374211]

22. Moher D, Liberati A, Tetzlaff J, Altman DG, PRISMA Group. Preferred reporting items for systematic reviews and meta-analyses: the PRISMA statement. Br Med J 2009 Jul 21;339:b2535 [FREE Full text] [doi: 10.1136/bmj.b2535] [Medline: 19622551$]$

23. McGowan J, Sampson M, Salzwedel DM, Cogo E, Foerster V, Lefebvre C. Press peer review of electronic search strategies: 2015 guideline statement. J Clin Epidemiol 2016 Dec;75:40-46 [FREE Full text] [doi: 10.1016/j.jclinepi.2016.01.021] [Medline: 27005575]

24. Ruco A, Dossa F, Tinmouth J, Llovet D, Kishibe T, Baxter NN. Social media and mobile health technology for cancer screening: a systematic review and meta-analysis protocol. BMJ Open 2020 Feb 05;10(2):e035411 [FREE Full text] [doi: 10.1136/bmjopen-2019-035411] [Medline: 32029500]

25. Sterne JA, Savovic J, Page MJ, Elbers RG, Blencowe NS, Boutron I, et al. RoB 2: a revised tool for assessing risk of bias in randomised trials. Br Med J 2019 Aug 28;366:14898. [doi: 10.1136/bmj.14898] [Medline: 31462531]

26. EPOC Resources for review authors. Cochrane Effective Practice and Organisation of Care (EPOC). 2017. URL: https:/ /tinyurl.com/2du6ffc4 [accessed 2020-09-15]

27. McGuinness LA, Higgins JPT. Risk-of-bias VISualization (robvis): An R package and Shiny web app for visualizing risk-of-bias assessments. Res Synth Methods 2020 Apr 26:55-61 [FREE Full text] [doi: 10.1002/jrsm.1411] [Medline: 32336025]

28. Higgins J, Thomas J, Chandler J, Cumpston M, Li T, Page M, et al. Cochrane Handbook for Systematic Reviews of Interventions Version 6.2. Chichester, UK: John Wiley \& Sons; 2019:1-728.

29. Arcas MM, Buron A, Ramis O, Esturi M, Hernández C, Macià F. [Can a mobile phone short message increase participation in breast cancer screening programmes?]. Rev Calid Asist 2014;29(4):188-196. [doi: 10.1016/j.cali.2014.02.003] [Medline: 25002239]

30. Baker DW, Brown T, Buchanan DR, Weil J, Balsley K, Ranalli L, et al. Comparative effectiveness of a multifaceted intervention to improve adherence to annual colorectal cancer screening in community health centers: a randomized clinical trial. JAMA Intern Med 2014 Aug;174(8):1235-1241. [doi: 10.1001/jamainternmed.2014.2352] [Medline: 24934845]

31. Chung IY, Kang E, Yom CK, Kim D, Sun Y, Hwang Y, et al. Effect of short message service as a reminder on breast self-examination in breast cancer patients: a randomized controlled trial. J Telemed Telecare 2015 Apr;21(3): 144-150. [doi: 10.1177/1357633X15571651] [Medline: 25697492]

32. Coronado GD, Rivelli JS, Fuoco MJ, Vollmer WM, Petrik AF, Keast E, et al. Effect of reminding patients to complete fecal immunochemical testing: a comparative effectiveness study of automated and live approaches. J Gen Intern Med 2018 Jan;33(1):72-78 [FREE Full text] [doi: 10.1007/s11606-017-4184-x] [Medline: 29019046]

33. Coronado GD, Thompson JH, Petrik AF, Nyongesa DB, Leo MC, Castillo M, et al. Patient-refined messaging for a mailed colorectal cancer screening program: findings from the PROMPT study. J Am Board Fam Med 2019;32(3):318-328 [FREE Full text] [doi: $10.3122 / \mathrm{jabfm} .2019 .03 .180275]$ [Medline: $\underline{31068396}$ ]

34. Erwin E, Aronson KJ, Day A, Ginsburg O, Macheku G, Feksi A, et al. SMS behaviour change communication and eVoucher interventions to increase uptake of cervical cancer screening in the Kilimanjaro and Arusha regions of Tanzania: a randomised, double-blind, controlled trial of effectiveness. BMJ Innov 2019 Jan;5(1):28-34 [FREE Full text] [doi: 10.1136/bmjinnov-2018-000276] [Medline: 31645991]

35. Firmino-Machado J, Varela S, Mendes R, Moreira A, Lunet N, SCAN-Cervical Cancer collaborators. A 3-step intervention to improve adherence to cervical cancer screening: the SCAN randomized controlled trial. Prev Med 2019 Jun;123:250-261. [doi: 10.1016/j.ypmed.2019.03.025] [Medline: 30936001]

36. Hagoel L, Neter E, Stein N, Rennert G. Harnessing the question-behavior effect to enhance colorectal cancer screening in an mHealth experiment. Am J Public Health 2016 Nov;106(11):1998-2004. [doi: 10.2105/AJPH.2016.303364] [Medline: 27631750]

37. Heydari E, Noroozi A. Comparison of two different educational methods for teachers' mammography based on the health belief model. Asian Pac J Cancer Prev 2015;16(16):6981-6986 [FREE Full text] [doi: 10.7314/apjcp.2015.16.16.6981] [Medline: 26514478]

38. Hirst Y, Skrobanski H, Kerrison RS, Kobayashi LC, Counsell N, Djedovic N, et al. Text-message Reminders in Colorectal Cancer Screening (TRICCS): a randomised controlled trial. Br J Cancer 2017 May 23;116(11):1408-1414 [FREE Full text] [doi: 10.1038/bjc.2017.117] [Medline: 28441381]

39. Huf S, Kerrison RS, King D, Chadborn T, Richmond A, Cunningham D, et al. Behavioral economics informed message content in text message reminders to improve cervical screening participation: two pragmatic randomized controlled trials. Prev Med 2020 Oct;139:106170. [doi: 10.1016/j.ypmed.2020.106170] [Medline: 32610059] 
40. Hwang KO, Ottenbacher AJ, Graham AL, Thomas EJ, Street RL, Vernon SW. Online narratives and peer support for colorectal cancer screening: a pilot randomized trial. Am J Prev Med 2013 Jul;45(1):98-107. [doi: 10.1016/j.amepre.2013.02.024] [Medline: 23790994]

41. Kerrison RS, Shukla H, Cunningham D, Oyebode O, Friedman E. Text-message reminders increase uptake of routine breast screening appointments: a randomised controlled trial in a hard-to-reach population. Br J Cancer 2015 Mar 17;112(6):1005-1010 [FREE Full text] [doi: 10.1038/bjc.2015.36] [Medline: 25668008]

42. Khademolhosseini F, Noroozi A, Tahmasebi R. The effect of health belief model-based education through Telegram instant messaging services on Pap smear performance. Asian Pac J Cancer Prev 2017 Aug 27;18(8):2221-2226 [FREE Full text] [doi: 10.22034/APJCP.2017.18.8.2221] [Medline: 28843259]

43. Lakkis NA, Atfeh AM, El-Zein YR, Mahmassani DM, Hamadeh GN. The effect of two types of sms-texts on the uptake of screening mammogram: a randomized controlled trial. Prev Med 2011 Oct;53(4-5):325-327. [doi:

10.1016/j.ypmed.2011.08.013] [Medline: 21871480]

44. Lee H, Ghebre R, Le C, Jang YJ, Sharratt M, Yee D. Mobile phone multilevel and multimedia messaging intervention for breast cancer screening: pilot randomized controlled trial. JMIR Mhealth Uhealth 2017 Nov 07;5(11):e154 [FREE Full text] [doi: 10.2196/mhealth.7091] [Medline: 29113961]

45. Miller DP, Denizard-Thompson N, Weaver KE, Case LD, Troyer JL, Spangler JG, et al. Effect of a digital health intervention on receipt of colorectal cancer screening in vulnerable patients: a randomized controlled trial. Ann Intern Med 2018 Apr 17;168(8):550-557 [FREE Full text] [doi: 10.7326/M17-2315] [Medline: 29532054]

46. Muller CJ, Robinson RF, Smith JJ, Jernigan MA, Hiratsuka V, Dillard DA, et al. Text message reminders increased colorectal cancer screening in a randomized trial with Alaska Native and American Indian people. Cancer 2017 Apr 15;123(8):1382-1389. [doi: 10.1002/cncr.30499] [Medline: 28001304]

47. Rashid RM, Mohamed M, Hamid ZA, Dahlui M. Is the phone call the most effective method for recall in cervical cancer screening?--results from a randomised control trial. Asian Pac J Cancer Prev 2013;14(10):5901-5904 [FREE Full text] [doi: 10.7314/apjcp.2013.14.10.5901] [Medline: 24289597]

48. Reiter PL, Katz ML, Bauermeister JA, Shoben AB, Paskett ED, McRee A. Increasing human papillomavirus vaccination among young gay and bisexual men: a randomized pilot trial of the outsmart HPV intervention. LGBT Health 2018 Jul;5(5):325-329 [FREE Full text] [doi: 10.1089/lgbt.2018.0059] [Medline: 29979642]

49. Richman AR, Maddy L, Torres E, Goldberg EJ. A randomized intervention study to evaluate whether electronic messaging can increase human papillomavirus vaccine completion and knowledge among college students. J Am Coll Health 2016;64(4):269-278. [doi: 10.1080/07448481.2015.1117466] [Medline: 26821923]

50. Sly JR, Miller SJ, Jandorf L. The digital divide and health disparities: a pilot study examining the use of short message service (SMS) for colonoscopy reminders. J Rac Ethn Health Dispar 2014 Jul 11;1(4):231-237. [doi: 10.1007/s40615-014-0029-z]

51. Vidal C, Garcia M, Benito L, Milà N, Binefa G, Moreno V. Use of text-message reminders to improve participation in a population-based breast cancer screening program. J Med Syst 2014 Sep;38(9):118-124. [doi: 10.1007/s10916-014-0118-x] [Medline: 25073694]

52. Wanyoro A, Kabiru E. Use of mobile phone short text message service to enhance cervical cancer screening at Thika Level 5 hospital, Kiambu County, Kenya: a randomised controlled trial. Res Obstet Gynaecol 2017;5(1):10-20. [doi: 10.5923/j.rog.20170501.03]

53. Wong MC, Ching JY, Huang J, Wong JC, Lam TY, Chan VC, et al. Effectiveness of reminder strategies on cancer screening adherence: a randomised controlled trial. Br J Gen Pract 2018 Sep;68(674):604-611 [FREE Full text] [doi: 10.3399/bjgp18X698369] [Medline: $\underline{\text { 30104327] }}$

54. Le D, Holt CL. CervixCheck: A spiritually-based text messaging intervention to promote cervical cancer awareness and Pap test screening intention among African-American women. J Health Commun 2018;23(9):842-853. [doi: 10.1080/10810730.2018.1528317] [Medline: $\underline{30300091]}$

55. Capık C, Gözüm S. The effect of web-assisted education and reminders on health belief, level of knowledge and early diagnosis behaviors regarding prostate cancer screening. Eur J Oncol Nurs 2012 Feb;16(1):71-77 [FREE Full text] [doi: 10.1016/j.ejon.2011.03.007] [Medline: 21530397]

56. Jessup DL, Glover IM, Daye D, Banzi L, Jones P, Choy G, et al. Implementation of digital awareness strategies to engage patients and providers in a lung cancer screening program: retrospective study. J Med Internet Res 2018 Feb 15;20(2):e52 [FREE Full text] [doi: 10.2196/jmir.8932] [Medline: 29449199]

57. Fornos LB, Urbansky KA, Villarreal R. Increasing cervical cancer screening for a multiethnic population of women in South Texas. J Cancer Educ 2014 Mar;29(1):62-68. [doi: 10.1007/s13187-013-0544-3] [Medline: 24170274]

58. Lee HY, Koopmeiners JS, Rhee TG, Raveis VH, Ahluwalia JS. Mobile phone text messaging intervention for cervical cancer screening: changes in knowledge and behavior pre-post intervention. J Med Internet Res 2014;16(8):e196 [FREE Full text] [doi: 10.2196/jmir.3576] [Medline: 25164545]

59. Lemos M, Rothes I, Oliveira F, Soares L. Raising cervical cancer awareness: analysing the incremental efficacy of Short Message Service. Health Edu J 2017 Sep 14;76(8):956-970 [FREE Full text] [doi: 10.1177/0017896917728306] 
60. Ganta V, Moonie S, Patel D, Hunt AT, Richardson J, Di John D, et al. Timely reminder interventions to improve annual Papanicolaou (Pap) smear rates among HIV-infected women in an outpatient center of southern Nevada: a short report. AIDS Care 2017 Dec;29(9):1099-1101. [doi: 10.1080/09540121.2017.1322677] [Medline: 28460538]

61. Lam TY, Wong MC, Ching JY, Chan V, Ng SK, Hui SN, et al. 210 - Effectiveness of Whatsapp reminder on compliance with colorectal cancer screening: a randomized controlled trial. Gastroenterology 2018 May;154(6):58-59. [doi: 10.1016/s0016-5085(18)30653-x]

62. Adler D, Abar B, Wood N, Bonham A. An intervention to increase uptake of cervical cancer screening among emergency department patients: results of a randomized pilot study. J Emerg Med 2019 Dec;57(6):836-843 [FREE Full text] [doi: 10.1016/j.jemermed.2019.07.021] [Medline: $\underline{31594738 \text { ] }}$

63. Romli R, Shahabudin S, Saddki N, Mokhtar N. Effectiveness of a health education program to improve knowledge and attitude towards cervical cancer and Pap smear: a controlled community trial in Malaysia. Asian Pac J Cancer Prev 2020 Mar 01;21(3):853-859 [FREE Full text] [doi: 10.31557/APJCP.2020.21.3.853] [Medline: 32212817]

64. Mahmud N, Doshi SD, Coniglio MS, Clermont M, Bernard D, Reitz C, et al. An automated text message navigation program improves the show rate for outpatient colonoscopy. Health Educ Behav 2019 Dec;46(6):942-946 [FREE Full text] [doi: 10.1177/1090198119869964] [Medline: 31431077]

65. Linde DS, Andersen MS, Mwaiselage J, Manongi R, Kjaer SK, Rasch V. Effectiveness of one-way text messaging on attendance to follow-up cervical cancer screening among human papillomavirus-positive Tanzanian women (Connected2Care): parallel-group randomized controlled trial. J Med Internet Res 2020 Apr 02;22(4):e15863 [FREE Full text] [doi: 10.2196/15863] [Medline: 32238335]

66. Lyson HC, Le GM, Zhang J, Rivadeneira N, Lyles C, Radcliffe K, et al. Social media as a tool to promote health awareness: results from an online cervical cancer prevention study. J Cancer Educ 2019 Aug;34(4):819-822. [doi: 10.1007/s13187-018-1379-8] [Medline: 29948924]

67. Key KV, Adegboyega A, Bush H, Aleshire ME, Contreras OA, Hatcher J. \#CRCFREE: Using social media to reduce colorectal cancer risk in rural adults. Am J Health Behav 2020 May 01;44(3):353-363. [doi: 10.5993/AJHB.44.3.8] [Medline: 32295683]

68. Rhoades H, Wenzel SL, Rice E, Winetrobe H, Henwood B. No digital divide? Technology use among homeless adults. J Soc Distress Homeless 2017 Mar 22;26(1):73-77 [FREE Full text] [doi: 10.1080/10530789.2017.1305140] [Medline: 31097900]

69. Brouwers MC, De Vito C, Bahirathan L, Carol A, Carroll JC, Cotterchio M, et al. What implementation interventions increase cancer screening rates? A systematic review. Implement Sci 2011 Sep 29;6:111 [FREE Full text] [doi: 10.1186/1748-5908-6-111] [Medline: 21958556]

70. Han CJ, Lee YJ, Demiris G. Interventions using social media for cancer prevention and management: a systematic review. Cancer Nurs 2018;41(6):19-31 [FREE Full text] [doi: 10.1097/NCC.0000000000000534] [Medline: 28753192]

71. Koïvogui A, Levi S, Finkler M, Lewkowicz S, Gombeaud T, Sabate JM, et al. Feasibility of encouraging participation in colorectal cancer screening campaigns by motivating people through the social network, Facebook. Colorectal Dis 2020 Oct;22(10):1325-1335. [doi: 10.1111/codi.15121] [Medline: 32397003]

\author{
Abbreviations \\ CRC: colorectal cancer \\ LMIC: low- and middle-income country \\ MeSH: Medical Subject Headings \\ mHealth: mobile health \\ OR: odds ratio \\ PRISMA: Preferred Reporting Items for Systematic Reviews and Meta-Analyses \\ PROSPERO: Prospective Register of Systematic Reviews \\ RCT: randomized controlled trial
}

Edited by $R$ Kukafka; submitted 31.12.20; peer-reviewed by L Guo, E Neter; comments to author 08.02.21; revised version received 15.02.21; accepted 21.06.21; published 30.07.21

Please cite as:

Ruco A, Dossa F, Tinmouth J, Llovet D, Jacobson J, Kishibe T, Baxter N

Social Media and mHealth Technology for Cancer Screening: Systematic Review and Meta-analysis

J Med Internet Res 2021;23(7):e26759

URL: https://www.jmir.org/2021/7/e26759

doi: $\underline{10.2196 / 26759}$

PMID: $\underline{34328423}$ 
(C)Arlinda Ruco, Fahima Dossa, Jill Tinmouth, Diego Llovet, Jenna Jacobson, Teruko Kishibe, Nancy Baxter. Originally published in the Journal of Medical Internet Research (https://www.jmir.org), 30.07.2021. This is an open-access article distributed under the terms of the Creative Commons Attribution License (https://creativecommons.org/licenses/by/4.0/), which permits unrestricted use, distribution, and reproduction in any medium, provided the original work, first published in the Journal of Medical Internet Research, is properly cited. The complete bibliographic information, a link to the original publication on https://www.jmir.org/, as well as this copyright and license information must be included. 